\title{
Theoretical Investigation of Structural and Electronic Properties of Ruthenium Azopyridine Complexes Dyes for Photovoltaic Applications by Using DFT and TD-DFT Methods
}

\author{
Nobel Kouakou N'guessan \\ Kafoumba Bamba \\ Ouattara Wawohinlin Patrice \\ Nahossé Ziao \\ Université Nangui Abrogoua
}

Laboratoire de Thermodynamique et Physico-Chimie du Milieu,

Doi: 10.19044/esj.2018.v14n21p424 URL:http://dx.doi.org/10.19044/esj.2018.v14n21p424

\begin{abstract}
In this work, a series of ruthenium azopyridine complexes was studied theoretically as a sensitizer in Dye Sensitized Solar Cells (DSSCs) using density functional theory (DFT) and time-dependent DFT (TD-DFT). These dyes derive from ruthenium azopyridine complex $\mathrm{RuCl}_{2}$ (Azpy) $)_{2}$ considered as the reference by grafting an anchoring group (-COOH). Hens, 4-Hmazpy, 5-Hmazpy and O-Hazpy ligands as well as Azpy were studied. For the four ligands, 20 isomers expected are studied. In order to explore their photoelectrical properties, the ground state and excited state properties of the isolated dyes have been calculated at B3LYP/LANL2DZ level. And the same work was done with the dye $\mathrm{RuCl}_{2}(5 \mathrm{Hmazpy})_{2}$ in interaction with titanium dioxide. Comparing to N3, the key parameters including the light harvesting efficiency (LHE), the electron injection driving force $\Delta G^{\text {inject }}$, the regeneration driving force $\Delta \mathrm{G}^{\text {regen }}$, the open circuit voltage $V_{\text {OC }}$, the life time $\tau$ and adsorption energy were all scrutinized in detail. It results from this calculation that the ruthenium azopyridine complexes can be used as sensitizer in DSSCs. This work has highlighted the predictive and the guiding role of the theoretical approach in the design and the conception of new dyes for solar cells.
\end{abstract}

Keywords: Solar cell, sensitizer, azopyridine, ruthenium, DFT. 


\section{Introduction}

The using of fossil fuels that is running out and the serious environmental pollution problem brought about have generated a great interest in the scientific community for the research of sustainable energy as well as for Dye sensitized solar cells (DSSCs) (Grätzel, 2009). DSSCs have been widely studied these last two decades because of their relative cheapest cost comparing to semiconductor p-n junction solar cells (Smedad, Bignozzi, \& Argazzi, 1994). In DSSCs, the photo sensitizer (Hagfeldt \& Gratzel, 1995) plays a crucial role in the conversion of light into electricity. It is thus the main element of the Grätzel cell (Grätzel, 2009). It fills entirely the function of electron pump. Its choice is determined by certain constraints: (i) the dye must have an anchoring group favouring a strong adsorption on the semiconductor's surface. (ii) The electronic transition responsible for the photovoltaic activity of the dye must display a wavelength comprised between 400 and $800 \mathrm{~nm}$. This transition must be both wide and high to cover the solar spectrum. (iii) Besides, to allow the electron injection the oxidation potential of the excited dye must be greater than the electronic affinity of the oxide. Otherwise said, the LUMO's energy of the dye must be above that of the semiconductor conduction band (CB) to ease the leap of electron from the HOMO to the conduction band. (iv) Moreover, the HOMO of the dye must be below the HOMO of the electrolyte $\left(\mathrm{I}^{-} / \mathrm{I}_{3}^{-}\right)$that is assumed to allow its regeneration (Nazeeruddin, Humphry-Baker, Liska, \& Grätzel, 2003). In DSSCs, the sensitizers have been considered as one of the most important components to show high performance, and therefore, many types of dyes have been investigated to improve the overall system's performance. Particularly, the derivative ruthenium dyes have been the main topic of the DSSCs development. Whereas the ruthenium (II) complexes, thanks to their wide absorption domain and their remarkable photo-physical properties, are among the most studied chromophores in the field of DSSCs during the last two decades. The ability of ruthenium complexes to absorb light in the visible range comes essentially from a charge transfer from the central metal atom to the peripheral ligands, following the excitation by a photon of appropriate energy through the Metal-to-Ligand Charge Transfer (MLCT) phenomenon. The structure of these complexes also favours the spatial separation of charges inside the chromophore. Indeed, when this one absorbs a photon then an electron migrates from the "heart" of the molecule towards the - $\mathrm{COOH}$ anchored group of the chromophore. This migration eases the injection of an electron to the $\mathrm{TiO}_{2}$. The central metal atom of these complexes therefore plays a crucial role in the photo-physical properties of the chromophores. But these can also be modulated by modifications of the ligands. DSSCs are based on the absorption of light by photosensitizers, and the most used and efficient dyes are based on the ruthenium polypyridyl complexes. However, the photo 
conversion yielded so far do not exceed 12\% (Das, Mondal, Mobin, \& Lahiri, 2009). Hence the need to develop new dyes to improve photo-conversion efficiency becomes paramount. Azopyridine ligands (Affi, Bamba, \& Ziao, 2015) are organic compounds consisting of a pyridine and an aromatic ring, linked to each other by an azo bond $\mathrm{N}=\mathrm{N}$. Figure 1 shows 2phenylazopyridine (Azpy) which is the reference and the most studied ligand (Krause \& Krause, 1980) (Anindya, Shie-Ming, \& Samaresh, 2000). Several types of substituents $\left(-\mathrm{CH}_{3},-\mathrm{COOH}\right.$...) can be linked to Azpy to make the complex much more active and selective (Figure 1). Here, the three ligands studied in addition to Azpy are 2-phenylazopyridine-4-methanoic acid (4Hmazpy), 2-phenylazopyridine-5-methanoic acid (5-Hmazpy) and 2phenylazopyridine-ortho-methanoic acid (O-Hmazpy). These compounds have a structure with a large system of $\pi$ conjugated bonds thereby offering particularly interesting perspectives for electronic applications. They stabilize the ruthenium atom at low oxidation state. Moreover, regarding their asymmetry structure, they are expected to perform with ruthenium five possible geometrical isomers (Figure 2) (Velders, et al., 2004).<smiles>Oc1ccccc1/N=N/c1ccccn1</smiles>

2-phenylazopyridine (Azpy)

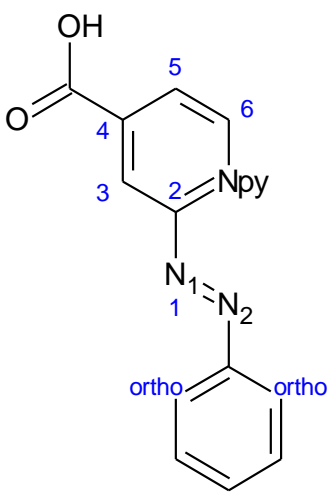
2-phenylazopyridine-4
-methanoic acid
(4-Hmazpy)<smiles>O=C(O)c1ccc(N=Nc2ccccc2O)nc1</smiles>

2-phenylazopyridine-5 -methanoic acid (5-Hmazpy)<smiles>O=C(O)c1cccc(O)c1N=Nc1ccccn1</smiles>

2-phenylazopyridineO-methanoic acid (O-Hmazpy)

Figure 1: Structures of 4 azopyridines ligands. 4-Hmazpy, 5-Hmazpy and O-Hmazpy are iso-electronical. The four ligands are expected to give out five isomers complexes $\operatorname{RuCl}_{2}(\mathrm{~L})_{2}$ regarding their asymmetry. Azpy and its relative five isomers have already been experimentally studied. Therefore, these results taken as reference will be used to predict those expected from complexes of 4-Hmazpy, 5-Hmazpy and O-Hmazpy ligands. 
<smiles></smiles>

$\operatorname{ctc}(\boldsymbol{\alpha}-\mathbf{C l})$<smiles>P=N[Te](Cl)(Cl)(Cl)N1CCNCC1</smiles>

$\operatorname{ccc}(\boldsymbol{\beta}-\mathbf{C I})$<smiles>NCCN=N[Te](Cl)(Cl)(Cl)N1CCNC1</smiles>

$\operatorname{cct}(\varepsilon-\mathbf{C l})$<smiles>[N][Te]1(Cl)(Cl)(Cl)C2NCCN1N2</smiles>

$\operatorname{tcc}(\gamma-\mathbf{C l})$<smiles>ClP1(Cl)(Cl)N=NCCN1</smiles>

$\mathrm{ttt}(\boldsymbol{\delta}-\mathrm{Cl})$

Figure 2: The five expected isomeric forms of azopyridine complexes $\mathrm{RuCl}_{2} \mathrm{~L}_{2}$. $\mathrm{L}$ represents azopyridine ligand. The three-letter code indicates the mutual $\operatorname{cis}(\mathrm{c})$ or trans $(\mathrm{t})$ orientation of the chlorides $(\mathrm{Cl})$, the pyridine $\left(\mathrm{N}_{\mathrm{py}}\right)$ and the azo nitrogen $\left(\mathrm{N}_{2}\right)$. The arcs represent the azopyridine ligands. Except $\beta-\mathrm{Cl}$ all isomers are $\mathrm{C}_{2}$ symmetrical (Velders, et al., 2004). The five Greek letters are deeply attached to the nature of isomers. Though four ligands are studied in this article with five isomers given by each ligand, we find it practical

to replace the $\mathrm{Cl}$ atom by the azopyridine ligand when it comes to make difference. Otherwise, $\mathrm{Cl}$ is kept when they are considered by group of the same nature regardless the ligand. For instance, $\beta-\mathrm{Cl}$ means that the four isomers $\beta$ are concerned.

All these azopyridine ruthenium complexes are admitted to display the MLCT transitions in the visible domain (Tiam-Huey, Tarun, Po-Ching, FenLing, \& Chung-Sun, 2003) (Changsaluk \& Hansongnern, 2005) (Campbell, Burrell, Officer D., \& Jolley, 2004) (Nobel, Bamba, Patrice, \& Ziao, 2017). The well-known and easily tuneable photo-physical, photochemical and electrochemical properties of these compounds make them excellent candidates for light harvesting systems and energy conversion devices. However, the complexes synthesized up to date do not carry any anchor group. Therefore, in this work, we aim to imagine, model and characterize new ruthenium-azopyridine chromophores with anchoring group to evaluate their photovoltaic properties in DSSC $_{\text {s }}$. Hitherto, the most widely and the most successful anchoring group used is the carboxylic acid (-COOH). It permits to ensure the effective adsorption of the dye on the surface. They also promote electronic coupling between the chromophores donors and the acceptors levels of the $\mathrm{TiO}_{2}$ semiconductor (O'Regan \& Graetzel, 1991). Furthermore, the anchoring of $\mathrm{TiO}_{2}$ was carried out by one or more functional groups and the selected ligands for this study derive from methylated azopyridine ligands (4mazpy, 5mazpy and tazpy) in which the methyl group is oxidized into a carboxylic group (Changsaluk \& Hansongnern, 2005) (N'guessan, Bamba, Ouattara, \& Ziao, 2017) (Hotze, et al., 2004). 
In addition, the density functional theory (DFT) and the time-dependent DFT are performed to elucidate the ability for these compounds to generate a photocurrent. Indeed, theoretical studies of the electronic and photochemical properties of photosensitizers are necessary to understand the relationship between structure, properties and performance to synthesize new molecules (Zhang C. , et al., 2009) (Ham \& Kim, 2010) (Zhang C. R., et al., 2010) (RuizAnchondo, Flores-Holguín, \& Glossman-Mitnik, 2010) (De Angelis, 2010).

\section{Methods}

\section{Theoretical background}

A measurement of the performance of DSSCs system is defined by the incident photon-to-current conversion efficiency (IPCE). It depends as well onto the light harvesting efficiency $\left(\Phi_{\mathrm{LHE}}\right)$, as on the electron injection efficiency $\left(\Phi_{\text {inject }}\right)$ and on the charge collection efficiency $\left(\Phi_{\mathrm{CC}}\right)$. So IPCE can be determined by the following expression:

$$
\text { IPCE }=\Phi_{\text {LHE }} \Phi_{\text {inject }} \Phi_{\mathrm{CC}}
$$

Through this equation, only both $\Phi_{\text {LHE }}$ and $\Phi_{\text {inject }}$ formulas are theoretically approximated. Furthermore, the efficiency of the electron density movement is related to both the optical absorption's intensity and the available electron transition. It could be estimated from the light harvesting efficiency (LHE) and the coefficient of effective electron transition (Qin \& Clark, 2007). The LHE must be both large and possible to maximize the induced photocurrent. The LHE is determined from the following expression:

Where $\mathrm{f}$ is the oscillator strength.

$$
\mathrm{LHE}=1-10^{-\mathrm{f}}
$$

The injection of an electron by photo induction in DSSCs can be defined as a charge transfer (Chen, et al., 2012) (Chen, Yang, \& Li, 2013) (Han, et al., 2013). Using the Marcus theory for electron transfer (Marcus, $1993)$, the $\Phi_{\text {inject }}$ can be approximated by the electron injection driving force $\Delta \mathrm{G}_{\text {inject }}$ (Fan, Tan, \& Deng, 2012). So, $\Delta \mathrm{G}_{\text {inject }}$ measures the electron injection rate and the current density in the short circuit $\left(\mathrm{J}_{\mathrm{SC}}\right)$ in DSSCs. Generally, $\Delta \mathrm{G}_{\text {inject }}$ and electron injection efficiency $\left(\Phi_{\text {inject }}\right)$ are closely related. Thus, a great absolute value of $\Delta \mathrm{G}_{\text {inject }}$ reveals a high electron injection efficiency. Moreover, $\Delta \mathrm{G}_{\text {inject }}$ can be evaluated according to the following relation (Preat, Michaux, Jacquemin, \& Perpete, 2009):

$$
\Delta G_{\text {inject }}=E_{O X}^{d y e *}-E_{C B}
$$

where $\mathrm{E}_{\mathrm{CB}}$ is the reduction potential (LUMO) of the semiconductor's conduction band. This redox potential is experimentally estimated at $-4.00 \mathrm{eV}$ (Asbury, Wang, Hao, Ghosh, \& Lian, 2001). Whereas $E_{O X}^{\text {dye* }}$ corresponds to the oxidation potential of the dye in the excited state. This quantity is 
determined from the redox potential of the dye's ground state $\left(E_{O X}^{d y e}\right)$ and the vertical transition energy corresponding to the maximum of absorption $E_{00}^{d y e}$ :

$$
E_{O X}^{d y e *}=E_{O X}^{d y e}-E_{00}^{d y e}
$$

$E_{O X}^{d y e}$ can be calculated by two approaches. The first approach is based on Koopmans' theorem. This theorem states that the energy of the oxidation potential of the ground state is expressed by the energy of the ionization potential $\left(E_{O X}^{\text {dye }}\right) \cdot E_{O X}^{\text {dye }}$ corresponds to the opposite of the $\mathrm{E}_{\mathrm{HOMO}}\left(\mathrm{IP}=-\mathrm{E}_{\mathrm{HOMO}}\right)$ (Koopmans, 1934). This approach finds its meaning in the context of density functional theory (Geerlings, De Proft, \& Langenaeker, 2003) (TorrentSucarrat, De Proft, Geerlings, \& Ayers, 2008). A second approach is based on the energy of the orbital which is at the origin of the electronic transition.

In addition, a more negative value of $\Delta \mathrm{G}_{\text {inject }}$ will translate a spontaneous injection of the electron from the dye to the semiconductor. Also, the research of Islam et al. showed that the electron injection efficiency in the excited state ( $\left.\Phi_{\text {inject }}\right)$ tends to 1 when $\Delta G_{\text {inject }}$ is greater than $0.2 \mathrm{eV}$ (Islam, Sugihara, \& Arakawa, 2003).Thermodynamically, the spontaneity of the process Charge transfer of the excited dye to the $\mathrm{TiO}_{2}$ conduction band requires LUMO energy value of dye higher than $\mathrm{E}_{\mathrm{CB}}=-4.0 \mathrm{eV}$. And $\mathrm{HOMO}$ energy value of the dye must be lower than the potential energy of electrolyte $I^{-} / I_{3}^{-}(-4.80 \mathrm{eV})$ (Cahen, Hodes, Graetzel, Guillermoles, \& Riess) is required for spontaneous regeneration of the charge.

Furthermore, the power conversion efficiency or energy conversion efficiency $(\eta)$ of a DSSCs can be calculated from the short-circuit current density $\left(\mathrm{J}_{\mathrm{SC}}\right)$, the open circuit voltage $\left(\mathrm{V}_{\mathrm{OC}}\right)$, the form factor $(f f)$ and intensity of the light Is as following:

$$
\eta=\frac{J_{S C} V_{O C} f f}{I_{S}}
$$

Herein, for better understanding the power of conversion's efficiency $(\eta)$ of the solar cells based on these sensitizers, we have analysed the Voc and Jsc of these dyes through discussing the crucial factors. While Jsc is determined by LHE, Voc is obtained in relation with $E_{\text {LUMO. According to the }}$ sensitized mechanism (electron injection from the excited dyes to the semiconductor's conduction band), single electron and single state approximation the following relationship is found (Zhang C. R., et al., 2010):

$$
e . V \mathrm{Oc}=E_{\mathrm{LUMO}}-E_{\mathrm{CB}}
$$

where $E_{\mathrm{CB}}$ is the energy of the semiconductor's conduction band edge, leading to Voc that may be obtained by applying the following formula:

$$
\text { Voc }=\left(E_{\mathrm{LUMO}}-E_{\mathrm{CB}}\right) / e
$$

It induces that the higher the $E_{\text {LUMO }}$ is, the larger the Voc will be. 
Furthermore, the efficiency of dye regeneration $\Delta G^{\text {regen }}$ (Shalabi A. S., El Mahdy, Assem, Taha, \& Soliman, 2014) can affect the rate's constant of redox process between the oxidized dye and the electrolyte. $\Delta \mathrm{G}_{\text {regen }}$ can be calculated by the following relation:

$$
\Delta \mathrm{G}^{\text {regen }}=E_{\text {OX }}^{\text {dye }} E_{\text {redox }}^{\text {electrolyte }}
$$

where $E_{O X}^{d y e}$ is the redox potential of the dye in the ground state, and $E_{\text {redox }}^{\text {electrolyte }}$ is the redox potential of the electrolyte. The ideal value of the redox potential of the electrolyte in vacuum is $3.5 \mathrm{eV}$ (Shalabi A. S., El Mahdy, Assem, Taha, \& Soliman, 2014).

Moreover, the life time of the excited state is an important factor for evaluating the efficiency of dye charge transfer (Shalabi A., El Mahdy, Taha, \& Soliman, 2015). In fact, the dye with a long life time of excited state will have an easy electron transfer. Therefore, the excited state lifetime of the dye can be evaluated via the following equation ( $\mathrm{Li}$, et al., 2015):

$$
\tau=\frac{1,499}{f \sigma^{2}}
$$

$\boldsymbol{\sigma}$ and $f$ respectively represent the transition wavenumber and the transition oscillator strength.

\section{Computational methods}

All calculations were performed using DFT methods with the Becke B3LYP 3-parameter hybrid functional (Becke, 1993) and the LanL2DZ dualzeta pseudopotential (Foresman \& Frisch, 1996). DFT is a quantum mechanical method used to investigate the electronic structure of many body systems. With this theory, the properties of a many-electron system can be determined. The use of the pseudopotential base Lanl2DZ is justified by its efficiency in treating the relativistic effects of the electrons of the ruthenium nucleus (Foresman \& Frisch, 1996). The geometrical optimization of the molecules has been performed beforehand to obtain the structure in the fundamental state. Then this stable configuration was confirmed by the calculation of the frequencies which revealed the absence of imaginary frequencies. The absorptions of the Electron spectra were evaluated using time-dependent density functional theory (TD-DFT) at the B3LYP / Lan12DZ level. Several theoretical studies on ruthenium complexes indicate that the DFT calculations with the effective pseudopotential Lanl2DZ agree well with the experimental results (Zhang C. R., et al., 2011) (Guo, et al., 2010). All these calculations were performed using the Gaussian 03 program package (Frisch, et al., 2004). 


\section{Results and Discussion \\ The Geometric structure}

The optimized molecular geometric structures of $\mathrm{RuCl}_{2}(4-\mathrm{Hmazpy})_{2}$ isomers are shown in Figure 3. Like the other studied dyes from 5-Hmazpy and O-Hmazpy ligands, these compounds have in common ruthenium as the atom donor of $\pi$-electron. Whereas the acceptor groups are known to be the azopyridine ligands which behave similarly referring to the nitrogen atoms responsible for the bonding. Hens, both azopyridines ligands bind the ruthenium atom by the lone pairs of the nitrogen atoms $\mathrm{N}_{\mathrm{py}}$ of the pyridine and $\mathrm{N}_{2}$ of the azo $\left(\mathrm{N}_{1}=\mathrm{N}_{2}\right)$.

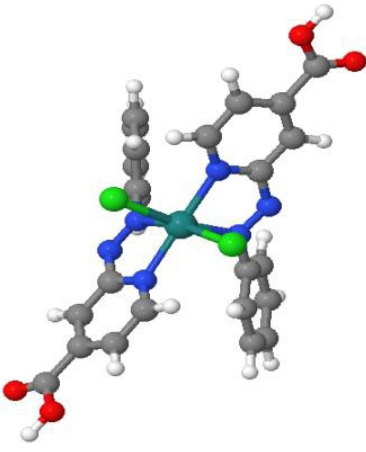

$\alpha-4$ Hmazpy

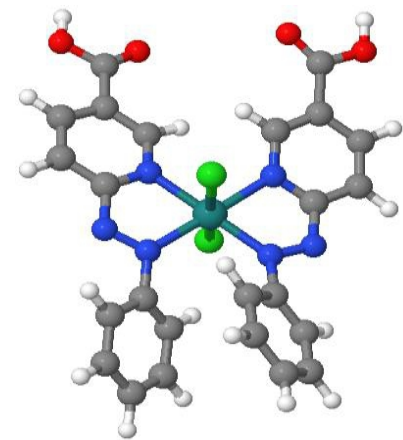

$\gamma-4$ Hmazpy

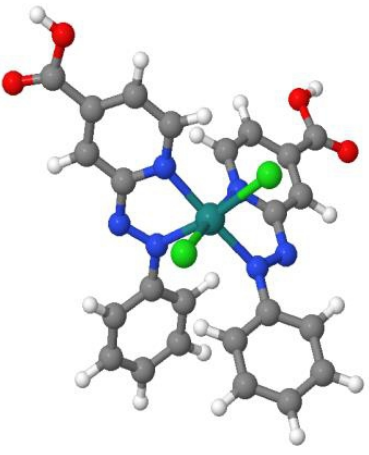

$\beta-4$ Hmazpy

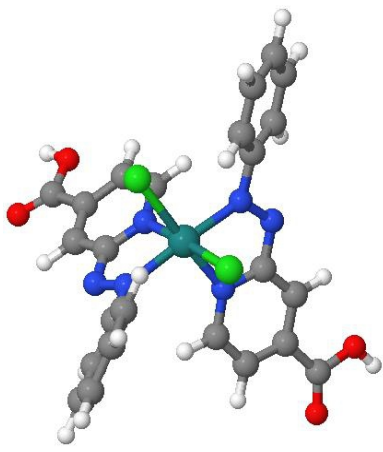

$\varepsilon$-4Hmazpy

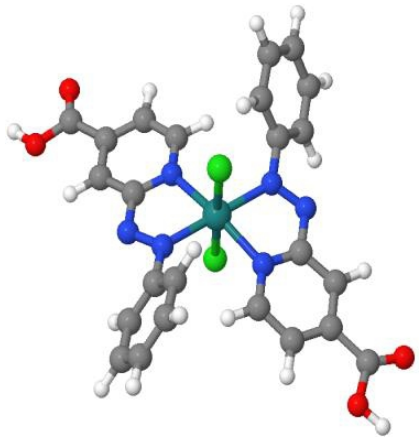

$\delta$-4Hmazpy

Figure 3: Optimized geometrical structures of isomers $\alpha-, \beta-, \varepsilon-, \gamma$ - and $\delta$ - $\mathrm{RuCl}_{2}(4 \mathrm{Hmazpy})_{2}$ at B3LYP/Lanl2DZ level in gas phase. 4Hmazpy stands for 2-phenylazopyridine-4methanoic acid. (The green coloured sphere: $\mathrm{Ru}$; the blue coloured spheres: $\mathrm{N}$; the larger dark coloured spheres: $\mathrm{C}$; the smaller white coloured spheres: $\mathrm{H}$, the red coloured spheres:

$\mathrm{O}$, the light green coloured sphere: $\mathrm{Cl}$ ).

Table 1 presents the optimized geometrical parameters of the complexes comparing to experimental data of $\mathrm{RuCl}_{2}(\mathrm{Azpy})_{2}$ (Velders, et al., 2004). Regarding their optimized geometries and data per bond, except $\beta-\mathrm{Cl}$ which presents an absence of symmetry, all other isomers $\alpha-\mathrm{Cl}, \gamma-\mathrm{Cl}, \delta-\mathrm{Cl}$ and $\varepsilon$-Cl display one data per bond indicating their $\mathrm{C}_{2}$ symmetry. Moreover, the 
values of bond lengths $\mathrm{Ru}-\mathrm{N}$ and $\mathrm{Ru}-\mathrm{Cl}$ are respectively comprised between 2.03-2.07 $\AA$; and 2.46-2.48 $\AA$. However, the lack of symmetry of $\beta-C l$ isomers is emphasised by double data observed in $\mathrm{Ru}-\mathrm{N}$ and $\mathrm{Ru}-\mathrm{Cl}$ bonds. Furthermore, the bond length values, as well as, the values of bond angles $\mathrm{X}$ $\mathrm{Ru}-\mathrm{X}(\mathrm{X}=\mathrm{Cl}$ or $\mathrm{N})$ are in the same order with the experimental data of $\mathrm{RuCl}_{2}$ (Azpy) $)_{2}$ complex (Velders, et al., 2004). It shows that the DFT method is reliable. From all compounds, only $\delta$-Cl isomers, keep the $\mathrm{Cl}-\mathrm{Ru}-\mathrm{Cl}$ angle without deformation at the ideal value of $180^{\circ}$. Whereas the remaining isomers undergo a serious modification of their $\mathrm{X}-\mathrm{Ru}-\mathrm{X}$ angles that doesn't also modify their symmetry regardless the nature of the ligand. Nevertheless, the slightly deformation noticed in each of the optimized octahedral structure must be attributed to the Yahn Teller effect (Shriver \& Atkins, 1999). 
Table 1: Comparison between the computational and experimental data for selected geometrical parameters, bond distances $(\AA)$ and angles $\left({ }^{\circ}\right)$ of

\begin{tabular}{|c|c|c|c|c|c|c|c|c|c|c|}
\hline & & & $\mathbf{N}_{1}=\mathbf{N}_{2}$ & Ru-N 2 & Ru-N $\mathbf{N}_{\mathbf{p y}}$ & Ru-Cl & Ru-Cl 2 & $\mathrm{Cl}_{1}-\mathrm{Ru}-\mathrm{Cl}_{2}$ & $\mathbf{N}_{p y}-\mathbf{R u}-\mathbf{N}_{\mathrm{py}}$ & $\mathbf{N}_{2}-\mathrm{Ru}-\mathrm{N}_{2}$ \\
\hline & \multirow{2}{*}{$\alpha$-Azpy } & Calc & 1.32 & 2.03 & 2.06 & 2.48 & 2.48 & 90.60 & 178.37 & 101.49 \\
\hline & & $\exp$ & 1.28 & 1.98 & 2.05 & 2.40 & 2.40 & 89.50 & 174.50 & 93.50 \\
\hline & \multirow[b]{2}{*}{$\beta$-Azpy } & Calc & $\begin{array}{l}1.32 \\
1.32\end{array}$ & $\begin{array}{l}2.02 \\
2.05\end{array}$ & $\begin{array}{l}2.05 \\
2.07\end{array}$ & 2.48 & 2.48 & 90.18 & 99.21 & 104.58 \\
\hline & & $\exp$ & $\begin{array}{l}1.29 \\
1.3\end{array}$ & $\begin{array}{l}1.96 \\
2.00\end{array}$ & $\begin{array}{l}2.02 \\
2.06\end{array}$ & 2.40 & 2.41 & 91.10 & 101.90 & 103.00 \\
\hline \multirow[t]{7}{*}{$\operatorname{RuCl}_{2}(\mathrm{Azpy})_{2}$} & \multirow{2}{*}{$\gamma$-Azpy } & Calc & 1.32 & 2.03 & 2.10 & 2.48 & 2.48 & 170.71 & 102.86 & 104.99 \\
\hline & & $\exp$ & 1.31 & 1.99 & 2.11 & 2.38 & 2.38 & 170.50 & 103.80 & 104.10 \\
\hline & \multirow{2}{*}{$\delta$-Azpy } & Calc & 1.31 & 2.06 & 2.10 & 2.51 & 2.49 & 180.00 & 167.53 & 178.58 \\
\hline & & $\exp$ & 1.28 & 2.02 & 2.06 & 2.38 & 2.38 & 180.00 & 180.00 & 180.00 \\
\hline & $\varepsilon$-Azpy & Calc & 1.32 & 2.05 & 2.06 & 2.49 & 2.49 & 94.10 & 93.58 & 169.48 \\
\hline & $\alpha$-4Hmazpy & Calc & 1.32 & 2.04 & 2.06 & 2.47 & 2.47 & 92.06 & 178.59 & 90.22 \\
\hline & $\beta-4$ Hmazpy & Calc & 1.32 & $\begin{array}{l}2.03 \\
2.03\end{array}$ & $\begin{array}{l}2.05 \\
2.06\end{array}$ & 2.47 & 2.47 & 91.01 & 99.41 & 104.74 \\
\hline \multirow[t]{4}{*}{$\mathrm{RuCl}_{2}(4 \mathrm{Hmazpy})_{2}$} & $\gamma$-4Hmazpy & Calc & 1.32 & 2.02 & 2.11 & 2.47 & 2.47 & 169.96 & 102.78 & 104.74 \\
\hline & $\delta$-4Hmazpy & Calc & 1.31 & 2.07 & 2.09 & 2.50 & 2.49 & 180.00 & 166.96 & 178.53 \\
\hline & $\varepsilon-4$ Hmazpy & Calc & 1.32 & 2.06 & 2.05 & 2.48 & 2.48 & 93.75 & 93.95 & 169.87 \\
\hline & $\alpha-5$ Hmazpy & Calc & 1.32 & 2.03 & 2.07 & 2.46 & 2.46 & 92.17 & 178.14 & 90.70 \\
\hline \multirow[t]{6}{*}{$\operatorname{RuCl}_{2}(5 \mathrm{Hmazpy})_{2}$} & $\beta-5$ Hmazpy & Calc & 1.33 & $\begin{array}{l}2.04 \\
2.02\end{array}$ & $\begin{array}{l}2.06 \\
2.08\end{array}$ & 2.47 & 2.46 & 89.91 & 99.18 & 104.53 \\
\hline & $\gamma-5$ Hmazpy & Calc & 1.32 & 2.03 & 2.11 & 2.47 & 2.47 & 169.95 & 102.79 & 104.92 \\
\hline & $\delta-5$ Hmazpy & Calc & 1.31 & 2.06 & 2.10 & 2.51 & 2.48 & 180.00 & 166.35 & 177.62 \\
\hline & $\varepsilon-5$ Hmazpy & Calc & 1.32 & 2.05 & 2.07 & 2.48 & 2.48 & 94.53 & 93.19 & 169.56 \\
\hline & $\alpha$-OHmazpy & Calc & 1.31 & 2.03 & 2.07 & 2.48 & 2.48 & 91.72 & 176.02 & 95.87 \\
\hline & $\beta$-OHmazpy & Calc & 1.32 & $\begin{array}{l}2.04 \\
2.02\end{array}$ & $\begin{array}{l}2.08 \\
2.06\end{array}$ & 2.47 & 2.48 & 88.61 & 101.06 & 101.06 \\
\hline \multirow[t]{3}{*}{$\mathrm{RuCl}_{2}(\mathrm{OHmazpy})_{2}$} & $\gamma$-OHmazpy & Calc & 1.32 & 2.01 & 2.13 & 2.47 & 2.47 & 164.32 & 106.20 & 101.46 \\
\hline & $\delta$-OHmazpy & Calc & 1.31 & 2.07 & 2.09 & 2.52 & 2.48 & 180.00 & 169.67 & 173.08 \\
\hline & $\varepsilon$-OHmazpy & Calc & 1.31 & 2.06 & 2.06 & 2.49 & 2.49 & 93.75 & 93.95 & 169.87 \\
\hline
\end{tabular}




\section{Free enthalpy of reaction}

Table 2 compares the variations of free enthalpy and the energetic level of the frontier molecular orbitals of each isomer $\mathrm{RuCl}_{2} \mathrm{~L}_{2}$ and $\mathrm{N} 3$. The recorded values of free enthalpy of reaction are all negative. This result indicates the spontaneity of the formation reaction of these compounds at $298.15 \mathrm{~K}$ with a pressure of $1 \mathrm{~atm}$. As well as the reference complex $\mathrm{RuCl}_{2}$ (Azpy), the $\mathrm{RuCl}_{2}$ (4Hmazpy) $)_{2}$ and $\mathrm{RuCl}_{2}$ (5Hmazpy) $)_{2}$ complexes present their $\alpha-\mathrm{Cl}$ isomers as the most stable because of their lowest free enthalpy values. However, in the case of the $\mathrm{RuCl}_{2}(\mathrm{OHmazpy})_{2}$ dyes, the most stable is assumed to be $\varepsilon$-OHmazpy isomer. Furthermore, except in the case of $\varepsilon$-OHmazpy where free enthalpy decreases in comparison with the reference $\varepsilon-\mathrm{RuCl}_{2}(\mathrm{Azpy})_{2}$, the substitution of formic acid in the Azpy ligand generally increases the free enthalpy of isomers. Therefore, we can assume that the substitution of hydrogen by the formic acid destabilizes the isomers contrary to $\varepsilon$-OHmazpy whose stability increases. Moreover, we can tell that the substitution performed on the pyridine ring of the ligand destabilizes more the complex than when phenyl ring is modified. Anyway, $\alpha$-Azpy remains the most stable complex.

Table 2: Free enthalpy (in kcal/mol) and frontiers molecular orbitals (in $\mathrm{eV}$ ) of $\alpha-\mathrm{Cl}, \beta-\mathrm{Cl}$, $\gamma-\mathrm{Cl}, \delta-\mathrm{Cl}$ and $\varepsilon-\mathrm{Cl}$ of the four complexes $\mathrm{RuCl}_{2}(\mathrm{Azpy})_{2}, \mathrm{RuCl}_{2}(4 \mathrm{Hmazpy})_{2}$,

$\mathrm{RuCl}_{2}(5 \mathrm{Hmazpy})_{2}, \mathrm{RuCl}_{2}(\mathrm{OHmazpy})_{2}$.

\begin{tabular}{ccccc}
\hline Dye & $\begin{array}{c}\mathbf{E}_{\mathbf{H O M O}} \\
(\mathbf{e V})\end{array}$ & $\mathcal{E}_{\mathbf{L U M O}}(\mathbf{e V})$ & $\Delta \mathbf{E}_{\mathbf{L}-\mathbf{H}}(\mathbf{e V})$ & $\Delta G(\mathrm{kcal} / \mathrm{mol})$ \\
\hline$\alpha$-Azpy & -5.554 & -3.333 & 2.221 & -16.989 \\
$\beta$-Azpy & -5.525 & -3.224 & 2.301 & -13.796 \\
$\gamma$-Azpy & -5.386 & -3.366 & 2.020 & -9.010 \\
$\delta$-Azpy & -5.229 & -3.429 & 1.800 & -10.110 \\
$\varepsilon$-Azpy & -5.402 & -3.363 & 2.039 & -10.889 \\
$\alpha-4$ Hmazpy & -5.752 & -3.686 & 2.066 & -13.290 \\
$\beta$-4Hmazpy & -5.841 & -3.618 & 2.223 & -10.350 \\
$\gamma-4$ Hmazpy & -5.72 & -3.78 & 1.940 & -8.129 \\
$\delta$-4Hmazpy & -5.568 & -3.826 & 1.742 & -7.342 \\
$\varepsilon-4 H$ mazpy & -5.742 & -3.77 & 1.972 & -7.875 \\
$\alpha-5$ Hmazpy & -5.736 & -3.787 & 1.949 & -10.437 \\
$\beta$-5Hmazpy & -5.828 & -3.69 & 2.138 & -9.221 \\
$\gamma-5$ Hmazpy & -5.72 & -3.78 & 1.940 & -5.531 \\
$\delta$-5Hmazpy & -5.566 & -3.933 & 1.633 & -5.129 \\
$\varepsilon-5 H m a z p y$ & -5.718 & -3.854 & 1.864 & -7.053 \\
$\alpha$ - OHmazpy & -5.672 & -3.469 & 2.203 & -12.883 \\
$\beta$ - OHmazpy & -5.720 & -3.46 & 2.260 & -9.282 \\
$\gamma$ - OHmazpy & -5.449 & -3.355 & 2.094 & -7.430 \\
$\delta$ - OHmazpy & -5.346 & -3.649 & 1.697 & -8.216 \\
$\varepsilon$ - OHmazpy & -5.570 & -3.440 & 2.130 & -13.405 \\
\hline N3 & -5.081 & -3.653 & 1.428 & - \\
\hline
\end{tabular}




\section{Frontier Molecular orbitals (FMOs)}

The energy levels of the highest occupied molecular orbital (HOMO) and the lowest unoccupied molecular orbital (LUMO), as well as the HOMOLUMO gaps are drawn in Figure 4. In addition, Table 2 compares the HOMO, LUMO and HOMO-LUMO gap values of the dyes with those of N3. The higher HOMO-LUMO gap is, the more difficult will be the transfer of electrons and therefore, the molecules will be hard and less active. However, the small HOMO-LUMO gap indicates an easy electrons transfer, which makes them soft and more active (Fukui, Theory of Orientation, 1975.) (Fukui, Yonezawa, \& Shingu, 1952). Hens, the small gap energy induces the highest efficiency of DSSC. In consequence, a small HOMO-LUMO gap is advantageous for absorbing a long wavelength (Bourouina, Rekhis, \& Trari, 2017). The recorded gap values reveal that the $\delta$ - $\mathrm{Cl}$ isomers are the most active. However, this reactivity is lower than N3's one that is known as the most active dye up today in DSSC and whose value $1.428 \mathrm{eV}$ is the lowest in Table 2. Moreover, Figure 4 illustrates the comparison of HOMO and LUMO energies of the dyes with the energy level of semiconductor $\mathrm{TiO}_{2}$ conduction band $(-4.00 \mathrm{eV})$ and the redox potential of the electrolyte $\mathrm{I}^{-} / \mathrm{I}_{3}{ }^{-}$(Cahen, Hodes, Graetzel, Guillermoles, \& Riess).

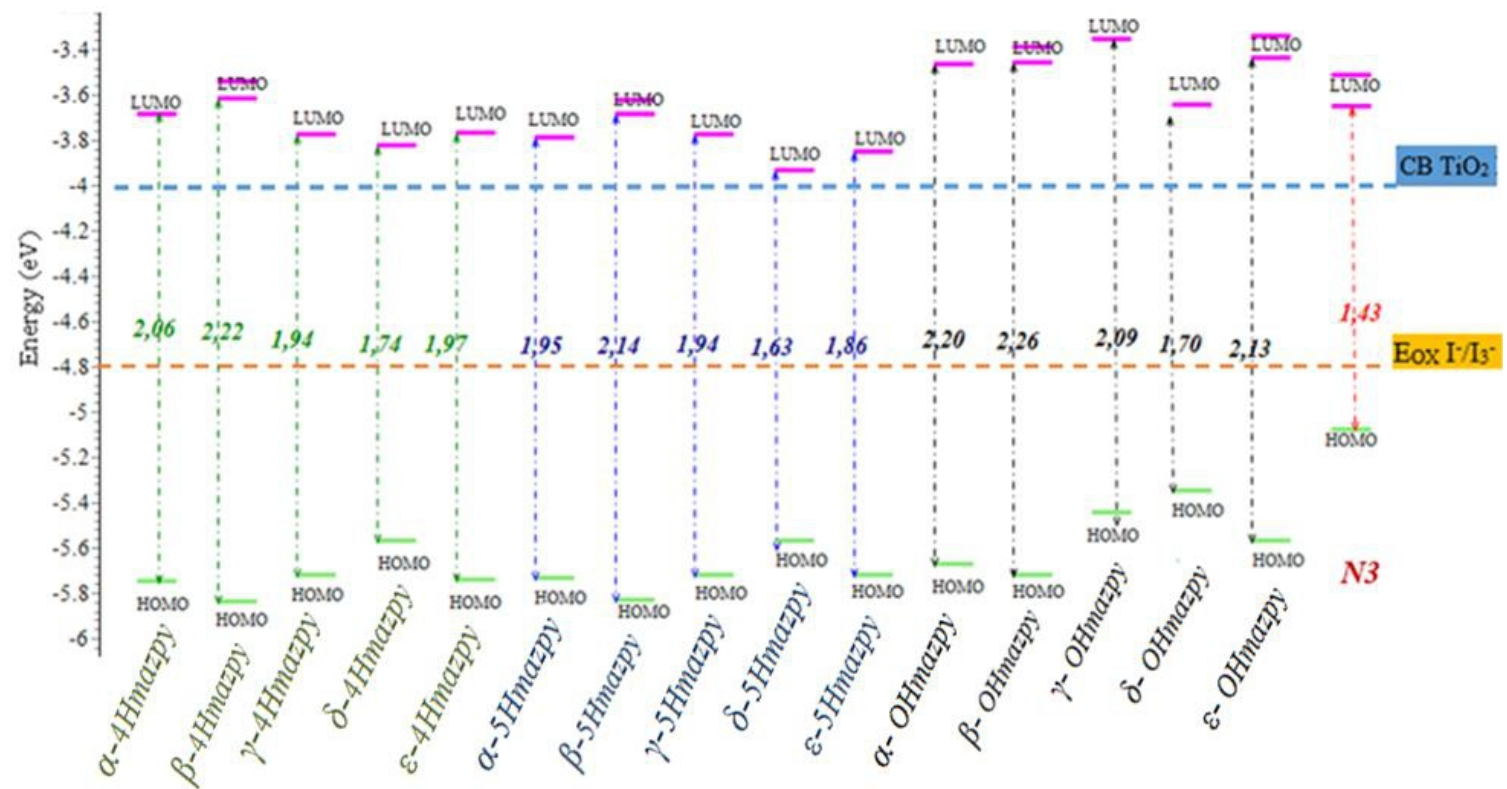

Figure 4: The energy levels of HOMO and LUMO, as well as HOMO-LUMO gap energy $(\mathrm{eV})$ of $\alpha-, \beta-, \gamma-, \delta$ - and $\varepsilon-\mathrm{RuCl}_{2}(\mathrm{iHmazpy})_{2}(\mathrm{i}=4,5$ and $\mathrm{O})$. The redox potential of $\mathrm{I}^{-} / \mathrm{I}_{3}{ }^{-}$ $(-4.80 \mathrm{eV})$ and energy level of conduction band $(\mathrm{CB})$ of the $\mathrm{TiO}_{2}$ surface $(-4.00 \mathrm{eV})$ are drawn respectively in orange and blue line.

The energy levels of the LUMO molecular orbitals far from the conduction band of the metal oxide could hamper the good injection of 
electrons into the semiconductor and favour certain recombination phenomena. The comparison of the energy levels of the different dyes with those of the other constituents of a DSSCs namely the $\mathrm{CB}$ of $\mathrm{TiO}_{2}$ and the electrolyte $I^{-} / I_{3}^{-}$shows a compatibility between them. Indeed, the LUMO of chromophores in all cases is located above the conduction band of $\mathrm{TiO}_{2}$ (Zhang C. , et al., 2009). In addition, the LUMO of the chromophores $\alpha$ 4Hmazpy, $\gamma$-4Hmazpy, $\delta$-4Hmazpy, $\varepsilon-4$ Hmazpy, $\alpha$-5Hmazpy, $\beta$-5Hmazpy, $\gamma$ 5 Hmazpy, $\delta$-5Hmazpy and $\varepsilon-5 \mathrm{Hmazpy}$ are lower than that of N3. Hence, these compounds would have an electron injection rate from the chromophore to the semiconductor better than that of $\mathrm{N} 3$. This result reveals their potential efficiency as photosensitisers. In addition, the examination of the HOMO value of the dyes indicates that the regeneration of the dyes by the electrolyte $I^{-} / I_{3}^{-}$could be optimal since the energy levels of the HOMO of the dyes are far lower than the oxidation potential of the $I^{-} / I_{3}^{-}$couple. In this case, the energy levels of this series of chromophores are in good agreement with the energy constraints imposed by the system.

Figure 5 shows the distribution of the isodensity surfaces of the Frontier molecular orbital (FMOs) of the studied compounds. These theoretical calculations show that there is a good spatial separation of the FOMs in all the cases. The HOMO of the dyes is distributed in the same way with a high electronic density on ruthenium atom. Also, LUMO and LUMO + 1 dyes are highly localized on their electro-accepting parts which are the azopyridine ligand and the formic acid function. Table 3 shows the contributions of ruthenium and azopyridine ligand respectively for HOMO and LUMO. The examination of the contribution of ruthenium to the HOMO of the complexes is between 44 and $61 \%$. And the contribution of the ligand to the LUMO is comprised between 83 and $95 \%$. Thus, the HOMOs and LUMOs of the dyes are assumed to correspond respectively to d orbital of metal and $\pi^{*}$ molecular orbital of ligand. Consequently, we can assume that the HOMO $\rightarrow$ LUMO transition is a metal ligand charge transfer (MLCT) of the $\mathrm{d} \rightarrow \pi^{*}$ type.

\section{Electronic excitations and absorption spectra}

From the ground-state geometric structure, the absorption spectra of the dyes were calculated by TD-DFT method at B3LYP / Lanl2DZ level in vacuum. The simulated absorption spectra are shown in Figure 6 and the corresponding absorption data are shown in Table 3 . These compounds absorb in the visible range $(400-800 \mathrm{~nm})$. The UV-visible absorbance spectra are characterized by two bands with different intensities. The transitions of higher energies are centred on the ligands. These transitions appear at wavelengths which are inferior to $500 \mathrm{~nm}$. These electronic transitions correspond to ligand to ligand charge transfer (LLCT) of $\pi \rightarrow \pi^{*}$ type. 
The transitions which are observed above $500 \mathrm{~nm}$ correspond to MLCT transitions from the metal to the ligand. These electronic transitions correspond to an electron transfer from the HOMOs orbitals characterized by the metal towards the LUMOs orbitals located on the ligand and the anchorage. The dyes $\alpha$-4Hmazpy, $\beta$-4Hmazpy, $\gamma$-4Hmazpy, $\delta$-4Hmazpy and $\varepsilon$-4Hmazpy respectively exhibit the maximum absorption of the MLCT transition at $662.808,699.847,624.29,543.984$ and $763.36 \mathrm{~nm}$. Concerning the dyes $\alpha$-5Hmazpy, $\beta$-5Hmazpy, $\gamma$-5Hmazpy, $\delta$-5Hmazpy and $\varepsilon$-5Hmazpy, they present their MLCT transitions respectively at 704.700, 711.004, $624.294,907.915$ and $800.109 \mathrm{~nm}$. For the $\alpha$-OHmazpy, $\beta$-OHmazpy, $\gamma$ OHmazpy, $\delta$-OHmazpy and $\varepsilon$-OHmazpy dyes, MLCT transitions of maximum energy are recorded at 624.106, 659.249, 607.055, 853.479 and $686.669 \mathrm{~nm}$, respectively. In the case of the $\delta$-Cl isomers, a broad MLCT band is observed for low energies of excitation. This can be explained by the flatness of the ligands in these compounds. This result is an advantage for their use as a sensitizer. 
-4-4mazpy

$\beta-4 H m a z p y$

L

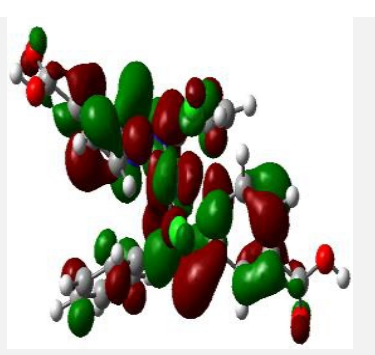

$\mathrm{H}$

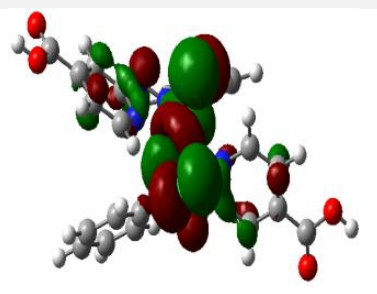

$\alpha-5$ Hmazpy

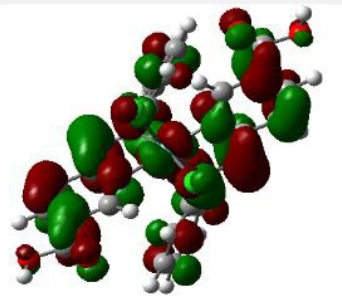

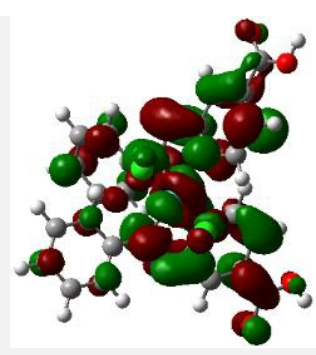

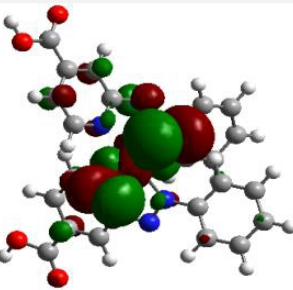

$\beta$-5Hmazpy

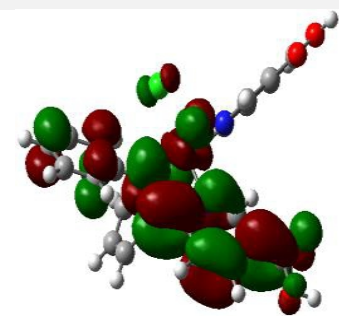

$\gamma-4$ Hmazpy
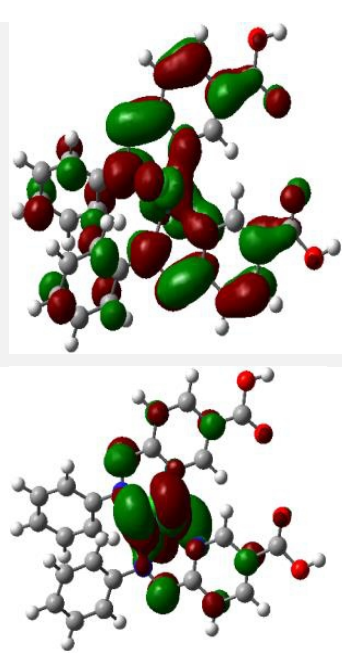

$\gamma-5$ Hmazpy

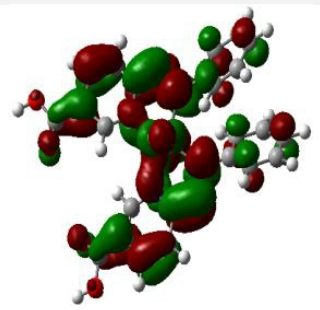

$\delta$-4Hmazpy
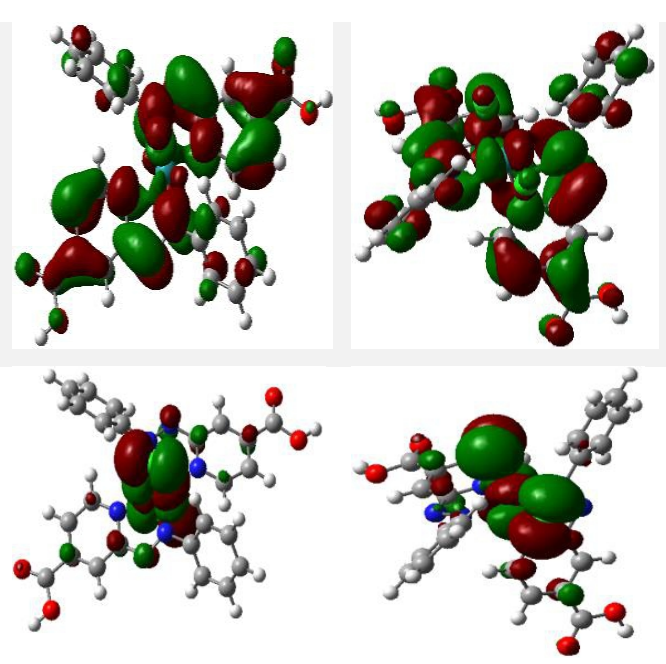

$\delta$-5Hmazpy

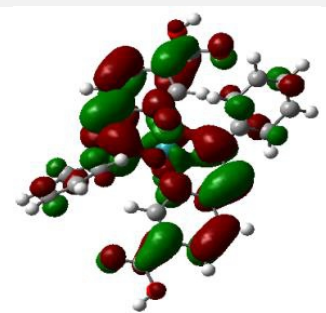

$\varepsilon-4$ Hmazpy

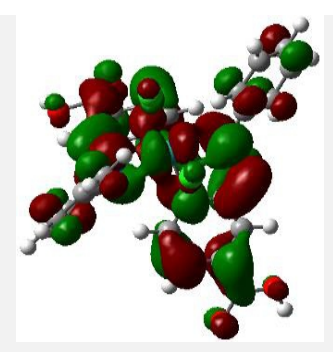

ع-5Hmazpy

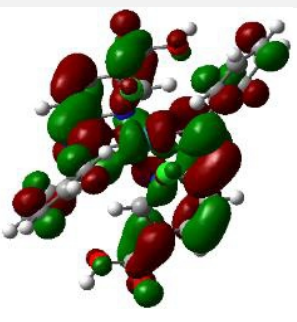


$\mathrm{H}$

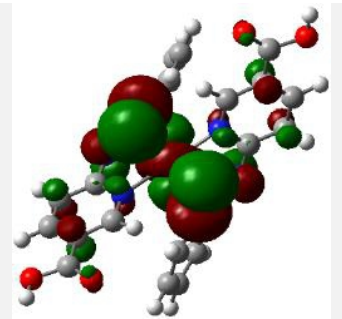

$\alpha$-OHmazpy

$\mathrm{L}$

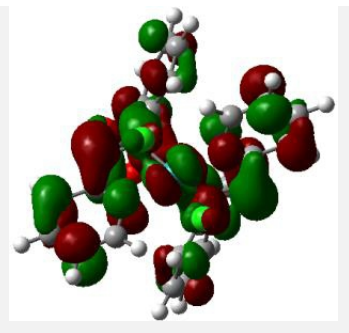

$\beta$-OHmazpy
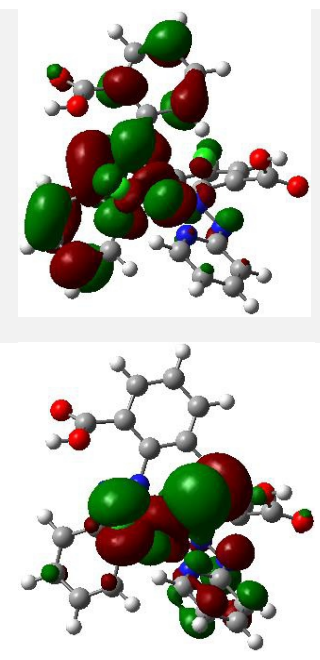

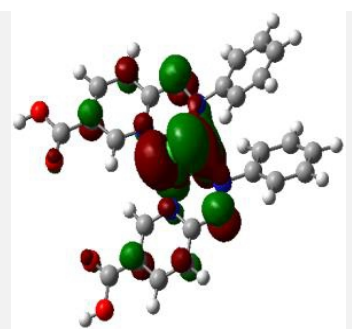

$\gamma$-OHmazpy
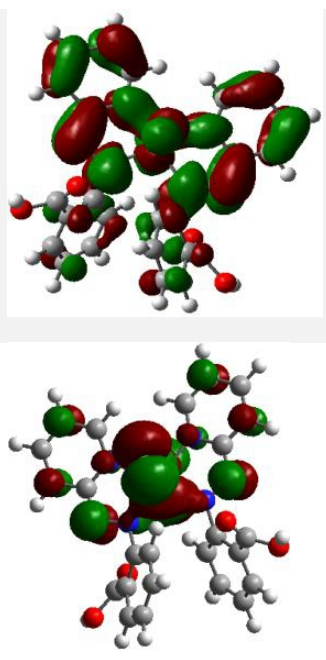

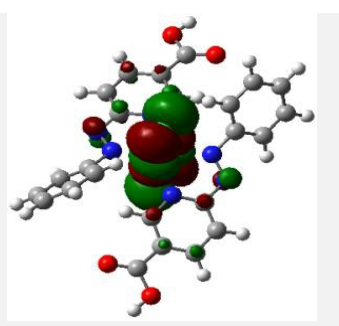

$\delta$-OHmazpy
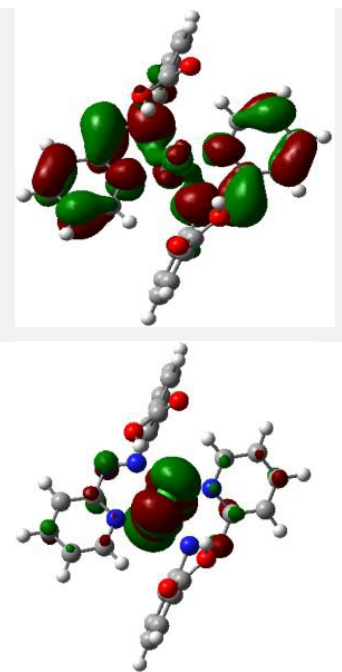

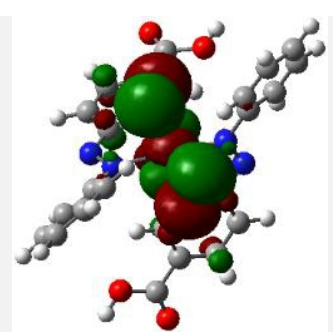

$\varepsilon$-OHmazpy
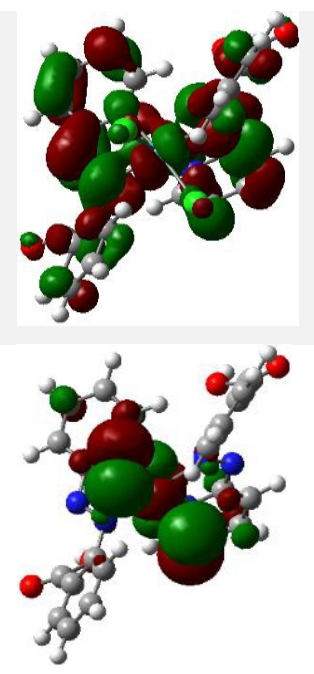

Figure 5: Representation of the frontier molecular orbital of $\alpha-, \beta-, \gamma-, \delta$ - and $\varepsilon-\mathrm{RuCl}_{2}(\mathrm{iHmazpy})_{2}(\mathrm{i}=4,5, \mathrm{O})$ determined by DFT method calculation, where $\mathrm{H}$ and $\mathrm{L}$ correspond respectively to HOMO and LUMO. 

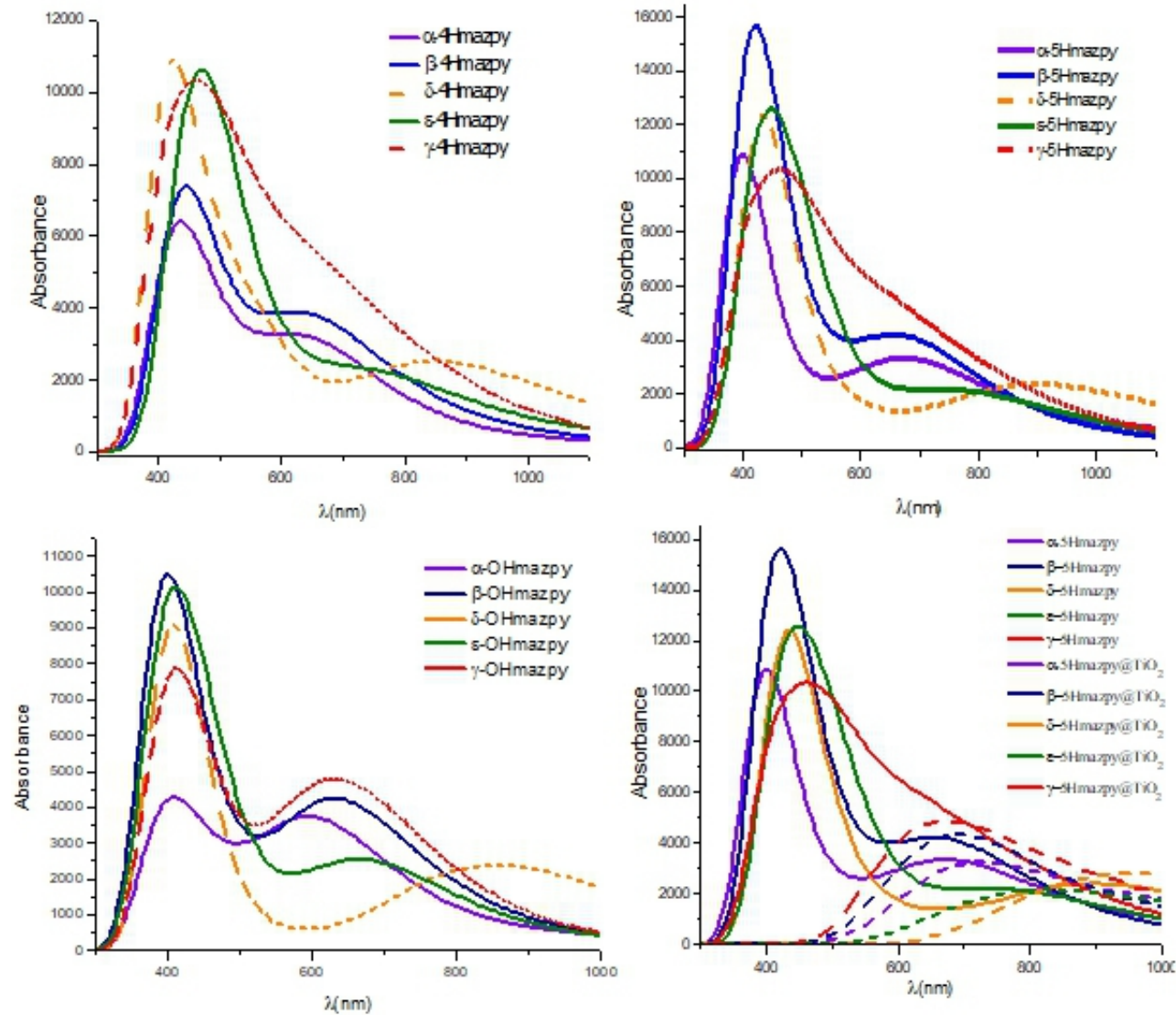

Figure 6: The UV-visible absorption spectra of isolated dyes $\alpha-, \beta-, \gamma-, \delta$ - and $\varepsilon$ $\mathrm{RuCl}_{2}(\mathrm{iHmazpy})_{2}(\mathrm{i}=4,5, \mathrm{O})$ and $\mathrm{RuCl}_{2}(5 \mathrm{Hmazpy})_{2}$ absorbed on $\mathrm{TiO}_{2}\left(5 \mathrm{Hmazpy} @ \mathrm{TiO}_{2}\right)$ and all calculated by using B3LYP/Lanl2dz level of theory in vacuum.

Table 3: Calculated transition properties of azopyridine dyes $\alpha-, \beta-, \gamma-, \delta$ - and $\varepsilon$ $\mathrm{RuCl}_{2}(\mathrm{iHmazpy})_{2}(\mathrm{i}=4,5, \mathrm{O})$ using B3LYP/Lanl2DZ in gas phase.

\begin{tabular}{|c|c|c|c|c|c|c|c|}
\hline \multirow[t]{2}{*}{ Dye } & \multicolumn{2}{|c|}{$\begin{array}{lll}\begin{array}{l}\text { composition } \\
\text { orbital }\end{array} & \text { of frontier } \\
\end{array}$} & \multirow[t]{2}{*}{$\Delta \mathbf{E}$} & \multirow[t]{2}{*}{$\begin{array}{l}\lambda \max \\
(\mathbf{n m})\end{array}$} & \multirow[t]{2}{*}{ f } & \multirow[t]{2}{*}{$\tau(\mathrm{ns})$} & \multirow[t]{2}{*}{$\begin{array}{l}\text { Electronic } \\
\text { transition }\end{array}$} \\
\hline & HOMO & LUMO & & & & & \\
\hline$\alpha-4 H m a z p y$ & $\mathrm{Ru}(47 \%)$ & L (89\%) & 1.871 & 662.808 & 0.058 & 113.49 & $\mathrm{H}-2 \rightarrow \mathrm{L}+153 \%$ \\
\hline$\beta-4 H m a z p y$ & $\mathrm{Ru}(44 \%)$ & $\mathrm{L}(85 \%)$ & 1.772 & 699.847 & 0.035 & 209.67 & $\mathrm{H}-1 \rightarrow \mathrm{L} \quad 44 \%$ \\
\hline$\gamma$-4Hmazpy & $\mathrm{Ru}(51 \%)$ & $\mathrm{L}(86 \%)$ & 1.986 & 624.294 & 0.097 & 60.23 & $\mathrm{H}-2 \rightarrow \mathrm{L} \quad 62 \%$ \\
\hline$\delta$-4Hmazpy & $\mathrm{Ru}(60 \%)$ & L (95\%) & 2.279 & 543.984 & 0.074 & 59.95 & $\mathrm{H} \rightarrow \mathrm{L}+263 \%$ \\
\hline ع-4Hmazpy & $\mathrm{Ru}(49 \%)$ & L (90\%) & 1.624 & 763.36 & 0.044 & 198.57 & $\mathrm{H}-2 \rightarrow \mathrm{L} 56 \%$ \\
\hline$\alpha-5$ Hmazpy & $\mathrm{Ru}(44 \%)$ & L (90\%) & 1.759 & 704.7 & 0.064 & 116.37 & $\mathrm{H}-2 \rightarrow \mathrm{L}+158 \%$ \\
\hline$\beta-5 H m a z p y$ & $\mathrm{Ru}(43 \%)$ & $\mathrm{L}(83 \%)$ & 1.744 & 711.004 & 0.037 & 204.76 & $\mathrm{H}-1 \rightarrow \mathrm{L}+1 \quad 49 \%$ \\
\hline$\gamma$-5Hmazpy & $\mathrm{Ru}(51 \%)$ & $\mathrm{L}(86 \%)$ & 1.986 & 624.294 & 0.097 & 60.23 & $\mathrm{H}-2 \rightarrow \mathrm{L} \quad 62 \%$ \\
\hline$\delta$-5Hmazpy & $\mathrm{Ru}(61 \%)$ & L (94\%) & 1.366 & 907.915 & 0.057 & 216.65 & $\mathrm{H}-1 \rightarrow \mathrm{L} 70 \%$ \\
\hline$\varepsilon-5 H$ mazpy & $\mathrm{Ru}(51 \%)$ & L (89\%) & 1.55 & 800.109 & 0.039 & 245.93 & $\mathrm{H}-2 \rightarrow \mathrm{L} 57 \%$ \\
\hline$\alpha$-OHmazpy & $\mathrm{Ru}(48 \%)$ & $\mathrm{L}(89 \%)$ & 1.987 & 624.106 & 0.066 & 88.43 & $\mathrm{H}-2 \rightarrow \mathrm{L}+156 \%$ \\
\hline
\end{tabular}




\begin{tabular}{|c|c|c|c|c|c|c|c|}
\hline$\beta$-OHmazpy & $\mathrm{Ru}(40 \%)$ & $\mathrm{L}(84 \%)$ & 1.881 & 659.249 & 0.04 & 162.82 & $\mathrm{H}-3 \rightarrow \mathrm{L} \quad 46 \%$ \\
\hline$\gamma$-OHmazpy & $\mathrm{Ru}(51 \%)$ & $\mathrm{L}(86 \%)$ & 2.042 & 607.055 & 0.08 & 69.08 & $\mathrm{H}-2 \rightarrow \mathrm{L} \quad 57 \%$ \\
\hline$\delta$-OHmazpy & $\mathrm{Ru}(60 \%)$ & $\mathrm{L}(95 \%)$ & 1.453 & 853.479 & 0.058 & 188.18 & $\mathrm{H}-1 \rightarrow \mathrm{L} 69 \%$ \\
\hline$\varepsilon$-OHmazpy & $\mathrm{Ru}(44 \%)$ & L (85\%) & 1.806 & 686.669 & 0.051 & 138.53 & $\mathrm{H}-2 \rightarrow \mathrm{L} 56 \%$ \\
\hline
\end{tabular}

\section{Photovoltaic properties}

Table 4 presents the theoretical characteristics of the performance of a dye. These parameters are the electron injection driving force $\left(\Delta G^{\text {inject }}\right)$, the efficiency of dye regeneration $\left(\Delta G^{\text {regen }}\right)$, the light harvesting efficiency LHE and the open circuit voltage $V_{O C}$. These parameters were obtained after DFT prediction followed by TD-DFT calculation at the same level (B3LYP / Lan12DZ).

Actually, as it is assumed that the higher value of the LHE maximizes the photo conversion, the following rank compares the LHE's values of the studied dyes.

$\delta$-5Hmazpy $>\delta$-4Hmazpy $>\gamma-4$ Hmazpy $>\gamma-5$ Hmazpy $>\beta-5$ Hmazpy $>\delta-$

OHmazpy $>\varepsilon-4$ Hmazpy $>\alpha-5$ Hmazpy $>\varepsilon-5$ Hmazpy $>\gamma-$ OHmazpy $>\beta-$ OHmazpy $>\varepsilon$-OHmazpy $>\alpha$-OHmazpy $>\alpha-4$ Hmazpy $>$ N3 $>\beta-4$ Hmazpy.

This sequence shows that, except $\beta$-4Hmazpy, all the studied dyes have a hole collection efficiency higher than that of $\mathrm{N} 3$ which equals to 0.121 . This result proves that these dyes maximize better the induced photocurrent than N3. In addition, $\delta$-5Hmazpy has the highest hole collection efficiency. However, regarding the ligand, we can assume that OHmazpy seems to hamper considerably $\mathrm{Ru}$ from producing energy.

Concerning the electron injection driving force, a more negative value of this parameter indicates a higher electron injection driving force in the excited state. The recorded values of the electron injection free enthalpy $\Delta G^{\text {inject }}$ allow to establish the following decreasing order of injection driving force:

$\delta$-OHmazpy $>\varepsilon-\mathrm{OHmazpy}>\beta$-OHmazpy $>\delta-4 \mathrm{Hmazpy}>\alpha-5 \mathrm{Hmazpy}>\delta$ -

5 Hmazpy $>\varepsilon-5$ Hmazpy $>\alpha-4$ Hmazpy $>\beta-5$ Hmazpy $>\beta-4$ Hmazpy $>\varepsilon-$

4 Hmazpy $>\gamma$-4Hmazpy $>\gamma-5$ Hmazpy $>\gamma$-OHmazpy $>\mathrm{N} 3>\alpha$-OHmazpy.

This evolution order of the injection rate reveals a great efficiency of electron injection of the studied dyes compared to N3. Indeed, the recorded values of $\Delta \mathrm{G}^{\text {inject }}$ except the one of $\alpha$-OHmazpy are more negative than that of $\mathrm{N} 3$. The $\delta$-OHmazpy dye has the highest absolute value of $\Delta \mathrm{G}^{\text {inject }}$ and will inject the electron as fast as possible into the semiconductor. In addition, the different values of the regeneration free enthalpy $\Delta \mathrm{G}^{\text {regen }}$ of the dyes presented in Table 4 are greater than those of N3. Here, it shows that these compounds will induce a higher speed constancy of the redox process between the oxidized dye and the electrolyte. Whereas the open circuit voltage $\left(\mathrm{V}_{\mathrm{OC}}\right)$ also 
theoretically determined, the values recorded for the dyes are shown through the decreasing order as follow:

$\gamma$-OHmazpy $>\varepsilon-O H m a z p y>\beta-O H m a z p y>\alpha-O H m a z p y>\beta-4 H m a z p y>\delta-$ OHmazpy $>\mathrm{N} 3>\alpha-4 \mathrm{Hmazpy}>\beta-5 \mathrm{Hmazpy}>\varepsilon-4 \mathrm{Hmazpy}>\gamma-4 \mathrm{Hmazpy}>\gamma-$

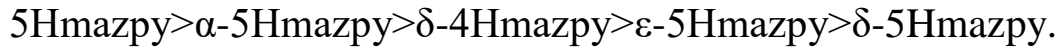

This order shows that $\beta$-4Hmazpy, $\alpha$-OHmazpy, $\beta$-OHmazpy, $\gamma-$ OHmazpy, $\delta$-OHmazpy and $\varepsilon$-OHmazpy have their values of open circuit voltages higher than that of $\mathrm{N} 3$. Therefore, the position of the $\mathrm{COOH}$ anchoring group in the ortho position on the phenyl of azopyridine is known to improve the $\mathrm{V}_{\mathrm{OC}}$. And the $\gamma$-OHmazpy dye is assumed to display the highest Voc. Furthermore, the life time of the excited state is recorded in Table 3. It is admitted that a long-life time keeps the dye longer in the cationic state, which allows a greater charge transfer (Li, et al., 2015). Therefore, as shown in Table 3 , the life time values of the excited state of the studied dyes are comprised between $59.95 \mathrm{~ns}$ and $245.93 \mathrm{~ns}$. These results show that the cationic ruthenium azopyridine complexes are stable for a long time. This result indicates a good charge transfer and an increasing photo conversion of DSSCs.

Table 4: Comparison of Performance Parameters of $\alpha-, \beta-, \gamma-, \delta$ - and $\varepsilon-\mathrm{RuCl}_{2}(\mathrm{iHmazpy})_{2}(\mathrm{i}=$ $4,5, \mathrm{O})$ and $\mathrm{N} 3$

\begin{tabular}{lllllllll}
\hline Colorants & $E_{\text {exc }}^{\text {dye }}$ & $f$ & LHE & $E_{O X}^{\text {dye }}$ & $E_{O X}^{\text {dyes }}$ & $\Delta G^{\text {regen }}$ & $\Delta G_{\text {TiO }}^{\text {inject }}$ & $V_{O C}$ \\
\hline$\alpha$-4Hmazpy & 2.971 & 0.061 & 0.131 & 5.752 & 2.781 & 20.132 & -1.219 & 0.314 \\
$\beta$-4Hmazpy & 2.915 & 0.043 & 0.094 & 5.841 & 2.926 & 20.444 & -1.074 & 0.382 \\
$\gamma$-4Hmazpy & 2.51 & 0.162 & 0.311 & 5.72 & 3.21 & 20.02 & -0.79 & 0.22 \\
$\delta$-4Hmazpy & 3.015 & 0.187 & 0.350 & 5.568 & 2.553 & 19.488 & -1.447 & 0.174 \\
$\varepsilon$-4Hmazpy & 2.805 & 0.102 & 0.209 & 5.742 & 2.937 & 20.097 & -1.063 & 0.23 \\
\hline$\alpha$-5Hmazpy & 3.159 & 0.098 & 0.202 & 5.736 & 2.577 & 20.076 & -1.423 & 0.213 \\
$\beta$-5Hmazpy & 3.002 & 0.131 & 0.260 & 5.828 & 2.826 & 20.398 & -1.174 & 0.31 \\
$\gamma$-5Hmazpy & 2.51 & 0.162 & 0.311 & 5.72 & 3.21 & 20.02 & -0.79 & 0.22 \\
$\delta$-5Hmazpy & 2.952 & 0.206 & 0.378 & 5.566 & 2.614 & 19.481 & -1.386 & 0.067 \\
$\varepsilon$-5Hmazpy & 2.977 & 0.084 & 0.176 & 5.718 & 2.741 & 20.013 & -1.259 & 0.146 \\
\hline$\alpha$-OHmazpy & 1.987 & 0.066 & 0.141 & 5.672 & 3.685 & 19.852 & -0.315 & 0.531 \\
$\beta$-OHmazpy & 3.229 & 0.076 & 0.161 & 5.720 & 2.491 & 20.020 & -1.509 & 0.540 \\
$\gamma$-OHmazpy & 2.042 & 0.080 & 0.168 & 5.449 & 3.407 & 19.072 & -0.593 & 0.645 \\
$\delta$-OHmazpy & 3.120 & 0.116 & 0.234 & 5.346 & 2.226 & 18.711 & -1.774 & 0.351 \\
$\varepsilon$-OHmazpy & 3.254 & 0.068 & 0.145 & 5.570 & 2.316 & 19.495 & -1.684 & 0.560 \\
\hline N3 & 1.610 & 0.056 & 0.121 & 5.081 & 3.471 & 17.784 & -0.529 & 0.347 \\
\hline
\end{tabular}

\section{Study of $\mathrm{RuCl} 2(5 \mathrm{Hmazpy}) 2$ dye isomers in interaction with $\mathrm{TiO} 2$ semiconductor}

The absorption of molecules on the surface of the semiconductor is an important factor in the conception of DSSCs. The functional groups, such as carboxyl groups, are essential for adsorbing the dye on the surface. From the 
structural and electronic points of view, they also induce a good cohesion of the organic and inorganic parts of the hybrid material. The chemisorption of the dyes was studied by DFT and TD-DFT methods at the same level of theory. The dye can be absorbed according to two common ways at the surface of titanium dioxide. While one is a single Ti-O monodentate binding mode, the other is accepted as a bidentate chelating structure mode (Xue-Feng, Jun, \& Guo-Jun, 2015). Figure 7 shows the optimized structures of $\mathrm{RuCl}_{2}$ (5Hmazpy $)_{2}$ dyes interacting with the semiconductor $\left(\mathrm{TiO}_{2}\right)_{4}$. These structures were obtained after geometry optimization and frequency calculation. The absence of imaginary frequency reveals that the ground state corresponds to the minimum of energy. The optimization of $\mathrm{RuCl}_{2}(5 \mathrm{Hmazpy})_{2} @ \mathrm{TiO}_{2}$ geometry showed that the bidentate-chelating configuration is a stable absorption configuration. Moreover, the absorption energy is determined by the following expression (Xue-Feng, Jun, \& Guo-Jun, 2015):

$$
E_{a b s}=E_{\left[d y e /\left(\mathrm{TiO}_{2}\right)_{4}\right]}-E_{\text {dye }}+E_{\left(\mathrm{TiO}_{2}\right)_{4}}
$$

Table 5. summarized the absorption energies and gap value of the dyes.

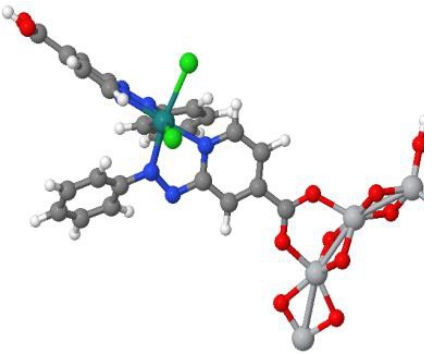

$\alpha-\mathrm{RuCl}_{2}(5 \mathrm{Hmazpy})_{2} @\left(\mathrm{TiO}_{2}\right)_{4}$

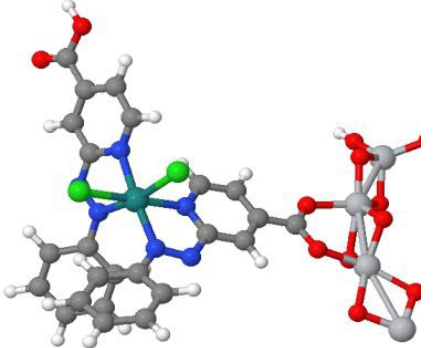

$\beta-\mathrm{RuCl}_{2}(5 \mathrm{Hmazpy})_{2} @\left(\mathrm{TiO}_{2}\right)_{4}$

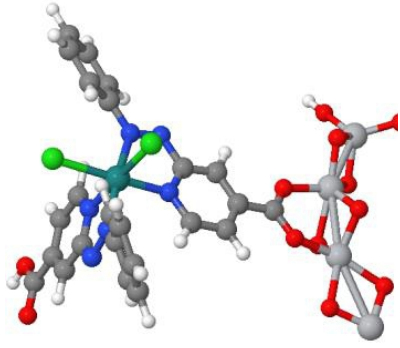

E- $\mathrm{RuCl}_{2}(5 \mathrm{Hmazpy})_{2} @\left(\mathrm{TiO}_{2}\right)_{4}$

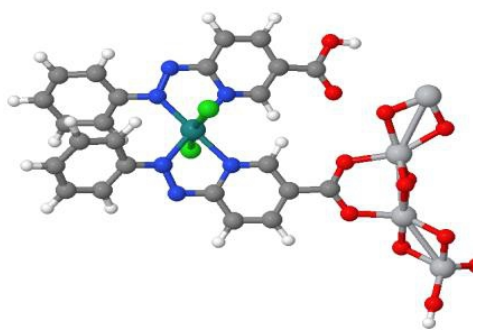

$\gamma-\mathrm{RuCl}_{2}(5 \mathrm{Hmazpy})_{2} @\left(\mathrm{TiO}_{2}\right)_{4}$

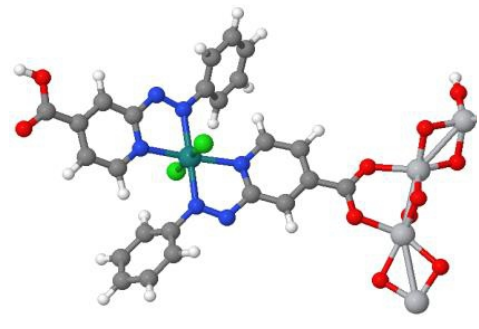

$\delta$ - $\mathrm{RuCl}_{2}(5 \mathrm{Hmazpy})_{2} @\left(\mathrm{TiO}_{2}\right)_{4}$

Figure 7 : Structures of azopyridine dye $\mathrm{RuCl}_{2}(5 \mathrm{Hmazpy})_{2}$ absorbed on $\left(\mathrm{TiO}_{2}\right)_{4}$ 
Table 5: Absorption energy parameters and gap value of azopyridine dye $\mathrm{RuCl}_{2}\left(5 \mathrm{Hmazpy}_{2}\right.$ absorbed on $\left(\mathrm{TiO}_{2}\right)_{4}$.

\begin{tabular}{|c|c|c|c|c|c|}
\hline & $E_{\text {dye (ua) }}$ & $E_{\left(\mathrm{TiO}_{2}\right)_{4}}$ (ua) & $E_{\left[d_{y / l} /\left(\mathrm{TiO}_{2}\right)_{4}\right]_{(\mathrm{ua})}}$ & $\begin{array}{c}\boldsymbol{E}_{\boldsymbol{a b s}} \\
(\mathrm{kcal} / \mathrm{mol})\end{array}$ & $\Delta \mathbf{E}_{\mathrm{L}-\mathrm{H}}$ \\
\hline a-5Hmazpy@ $\mathrm{TiO}_{2}$ & -1678.072 & -836.007 & -2513.580 & 313.629 & 1.918 \\
\hline$\beta-5$ Hmazpy@ $\mathrm{TiO}_{2}$ & -1678.071 & -836.007 & -2513.577 & 314.278 & 2.124 \\
\hline$\gamma$-5Hmazpy@ $\mathrm{TiO}_{2}$ & -1678.066 & -836.007 & -2513.573 & 314.382 & 1.929 \\
\hline 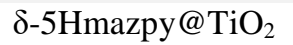 & -1678.064 & -836.007 & -2513.555 & 323.850 & 1.559 \\
\hline ع-5Hmazpy@ $\mathrm{TiO}_{2}$ & -1678.067 & -836.007 & -2513.575 & 313.515 & 1.851 \\
\hline
\end{tabular}

The negative energy values of the $\mathrm{RuCl}_{2}(5 \mathrm{Hmazpy})_{2} @ \mathrm{TiO}_{2}$ systems show that the absorption of the dyes on the surface of the titanium dioxide is possible at $298.15 \mathrm{~K}$ and at the pressure of $1 \mathrm{~atm}$. The absorption energies of the dyes are between 313.515 and $323.850 \mathrm{kcal}^{\mathrm{mol}}{ }^{-1}$. This result indicates a great absorption of dyes on the surface of titanium dioxide. The decreased order of $E_{a b s}$ for dyes $\mathrm{RuCl}_{2}(5 \mathrm{Hmazpy})_{2}$ is established as follows:

$\delta$-5Hmazpy@ $\mathrm{TiO}_{2}>\quad \gamma$-5 Hmazpy@ $\mathrm{TiO}_{2}>\beta-5 \mathrm{Hmazpy} @ \mathrm{TiO}_{2}>\quad \alpha$ 5Hmazpy@ $\mathrm{TiO}_{2}>\varepsilon-5 \mathrm{Hmazpy@ \textrm {TiO } _ { 2 }}$

This order shows that the dyes $\gamma-5$ Hmazpy and $\delta$-5Hmazpy are the most absorbed on the surface of the semiconductor. In addition, the $\delta$ 5 Hmazpy isomer is particularly by far the most adsorbed. This result indicates that the trans configuration of dyes is advantageous for good absorption. Furthermore, the calculated distances of all isomers $\mathrm{RuCl}_{2}(5 \mathrm{Hmazpy})_{2}$ between the carboxylic oxygen atoms and the $\left(\mathrm{TiO}_{2}\right)_{4}$ cluster (Ti-O bond lengths) are comprised between 1.95 and $2.08 \AA$. As far as the energy gap value of the systems is concerned, they permit to establish the following order:

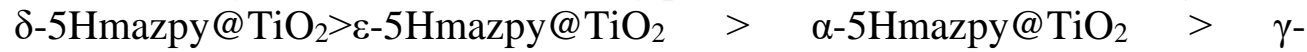
5Hmazpy@ $\mathrm{TiO}_{2}>\beta-5 \mathrm{Hmazpy@ \textrm {TiO } _ { 2 }}$

This order shows that the $\delta$-5Hmazpy@ $\mathrm{TiO}_{2}$ system is the most active and will therefore be the most photosensitive. Moreover, Figure 8 illustrates the frontier molecular orbitals of the $5 \mathrm{Hmazpy} @ \mathrm{TiO}_{2}$ system. The iso-density surfaces distribution shows that the HOMO is centred on metal and chlorine. While LUMO, LUMO + 1 and LUMO + 2 are localized on the azopyridine ligand and the semiconductor. This surface separation is favourable for the transfer of electrons from the dye to the semiconductor. The theoretical spectra of the 5Hmazpy@ $\mathrm{TiO}_{2}$ systems have a single absorption band which is above $500 \mathrm{~nm}$. This band is constituted by the electronic transitions corresponding to the electron transfer between the dye and the semiconductor (Figure 6). The $\delta$-5Hmazpy@ $\mathrm{TiO}_{2}$ system presents a broad absorption band in visible region. This property is an advantage for an efficient conversion of solar energy. 
$\alpha-5 \mathrm{Hmazpy} @\left(\mathrm{TiO}_{2}\right)_{4} \quad \beta-5 \mathrm{Hmazpy} @\left(\mathrm{TiO}_{2}\right)_{4} \quad \gamma-5 \mathrm{Hmazpy} @\left(\mathrm{TiO}_{2}\right)_{4} \quad \delta-5 \mathrm{Hmazpy} @\left(\mathrm{TiO}_{2}\right)_{4} \quad \varepsilon-5 \mathrm{Hmazpy} @\left(\mathrm{TiO}_{2}\right)_{4}$
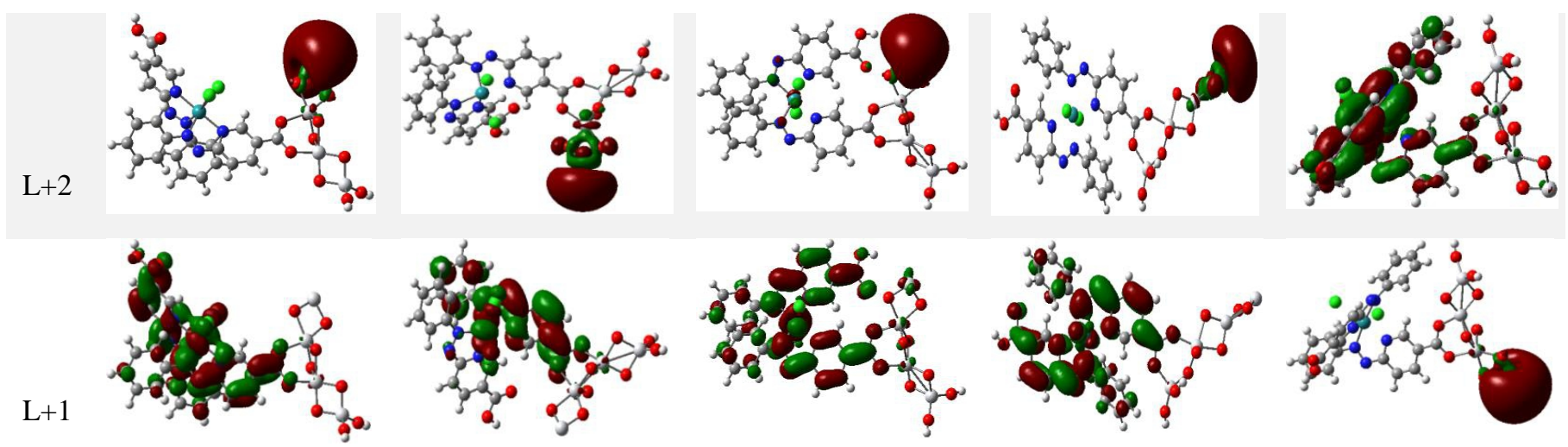

$\mathrm{L}$
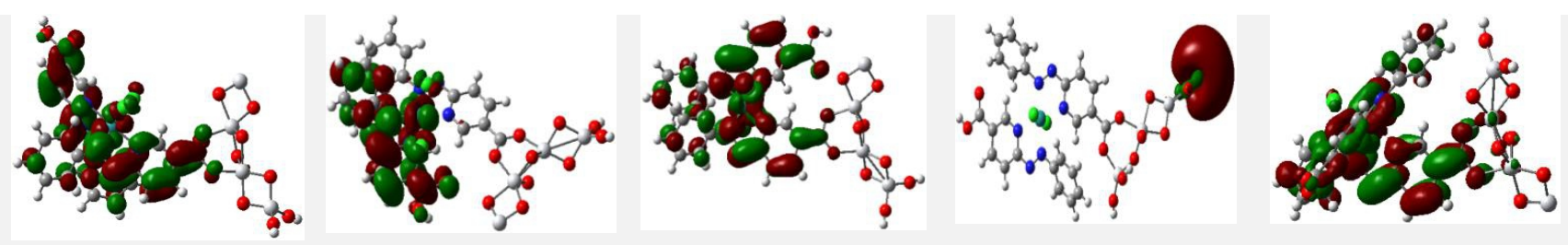

H
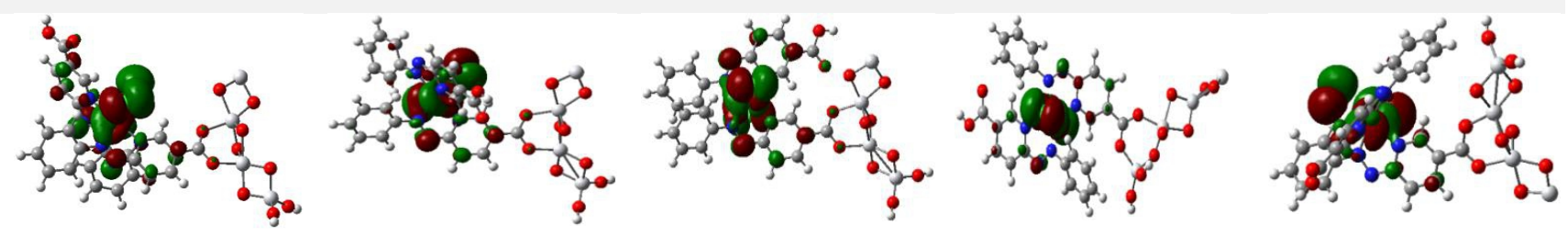

Figure 8 : Frontier molecular orbitals of $\mathrm{RuCl}_{2}(5 \mathrm{Hmazpy})_{2}$ absorbed on $\left(\mathrm{TiO}_{2}\right)_{4}$. 


\section{Conclusion}

In this work, electronic structure, absorption spectra, and photo physical properties of a series of azopyridine ruthenium complexes as photosensitizers for DSSCs applications were investigated by DFT and TDDFT methods. The results indicate that the anchoring group grafting $(-\mathrm{COOH})$ does not influence the structure of the reference $\mathrm{RuCl}_{2}(\text { azpy })_{2}$ complex. The modelled complexes are stable at the temperature of $298 \mathrm{~K}$ and the pressure of $1 \mathrm{~atm}$. The recorded gap values reveal that the $\delta$-iHmazpy $(\mathrm{i}=4,5, \mathrm{O})$ isomers are the most active of the studied dyes. The energy levels of HOMO and LUMO of dyes are in good agreement with the energy constraints imposed by the DSSCs system. Moreover, the study of the frontier molecular orbitals reveals that HOMO and LUMO are respectively localized on the metal and the ligand which is favourable to the MLCT transitions. This is confirmed by the theoretical absorption spectra of these isolated complexes which present MLCT transitions beyond $500 \mathrm{~nm}$. In the case of the $\delta$ isomers, a broad MLCT band is observed which makes them the most photosensitive compounds. In addition, the photovoltaic properties of the studied compounds, namely the light harvesting efficiency LHE, the free electron injection enthalpy $\Delta \mathrm{G}^{\text {inject }}$, the free regeneration enthalpy $\Delta \mathrm{G}^{\text {regen }}$ and the open circuit voltage $\left(\mathrm{V}_{\mathrm{OC}}\right)$ were determined and compared, to those of N3. It results from this study that in exception for $\beta-4 \mathrm{Hmazpy}$, these compounds have a hole collection efficiency higher than those of N3. Also, these compounds have a higher electron injection efficiency $\left(\Delta \mathrm{G}^{\text {inject }}\right)$ compared to $\mathrm{N} 3$ and can be regenerated by the $\mathrm{I}^{-}$ $/ \mathrm{I}_{3}{ }^{-}$electrolyte. Furthermore, the $\beta$-4Hmazpy, $\alpha$-OHmazpy, $\beta$-OHmazpy, $\gamma$ OHmazpy, $\delta$-OHmazpy and $\varepsilon$-OHmazpy dyes have their open circuit voltages greater than those of the N3. In addition, the ruthenium azopyridine complexes are stable for a long time in the cationic state. Finally, the investigation of the dyes $\mathrm{RuCl}_{2}(5 \mathrm{Hmazpy})_{2}$ in interaction with the $\mathrm{TiO}_{2}$ semiconductor shows that these systems are stable in a bidentate chelating structure mode. In sum, we can assume that the azopyridine complexes of ruthenium can be exploited as photosensitizers in DSSCs.

\section{References:}

1. Affi, S. T., Bamba, K., \& Ziao, N. (2015). computational characterization of organometallic ligands coordinating metal: case of azopyridine ligands. Journal of Theoretical and Computational Chemistry, 14.

2. Anindya, D., Shie-Ming, P., \& Samaresh, B. (2000). Chemistry of 2(phenylazo)pyridine complexes of osmium : Synthèsis, characterization and reactivities. Pohyhedron, 19, 1227, 1232.

3. Asbury, J. B., Wang, Y. Q., Hao, E., Ghosh, H. N., \& Lian, T. (2001). Evidences of hot excited state electron injection from sensitizer 
molecules to $\mathrm{TiO} 2$ nanocrystalline thin films. Res. Chem. Intermed., 27, 393-406.

4. Becke, A. D. (1993). A New Mixing of Hartree-Fock and Local Density-Functional Theories. The Journal of Chemical Physics, 98, 1372-1377.

5. Bourouina, A., Rekhis, M., \& Trari, M. (2017). DFT/TD-DFT study of ruthenium bipyridyl-based dyes with a chalcogen donor $(X=S, S e$, Te), for application as dye-sensitized solar cells. polyhedron, 30110-9.

6. Cahen, D., Hodes, G., Graetzel, M., Guillermoles, J. F., \& Riess, I. J. (s.d.). Nature of Photovoltaic Action in Dye-Sensitized Solar Cells. Phys. Chem. B, 104, 2053-2059.

7. Campbell, W. M., Burrell, A. K., Officer D., L., \& Jolley, K. W. (2004). Porphyrins as Light Harvesters in the Dye-Sensitised TiO2 Solar Cell. Coordination Chemistry Reviews, 248(13-14), 1363 1379.

8. Changsaluk, U., \& Hansongnern, K. (2005). Dichlorobi (5methyl-2(phenylazo)pyridine) ruthénium (II) complex : charaterisation and NMR sepectroscopy. SongKlanakain J. Sci Technol, 739-749.

9. Chen, J., Bai, F. Q., Wang, J., Hao, L., Xie, Z. F., Pan, Q. J., \& Zhang, H. X. (2012). Theoretical studies on spectroscopic properties of ruthenium sensitizers absorbed to $\mathrm{TiO} 2$ film surface with connection mode for DSSC. Dyes Pigm., 94, 459-468.

10. Chen, S. L., Yang, L. N., \& Li, Z. S. (2013). How to design more efficient organic dyes for dye sensitized solar cells? Adding more sp2hybridized nitrogen in the triphenylamine donor. J. Power Sources, 223, 86-93.

11. Das, D., Mondal, T. K., Mobin, S. M., \& Lahiri, G. K. (2009). "Sensitive Valence Structures of $[(\operatorname{Pap})(2) \mathrm{Ru}(\mathrm{Q})](\mathrm{N})(\mathrm{N}=+2,+1,0$,1,-2) with Two Different Redox Noninnocent Ligands, Q=3,5-Di-TertButyl-N-Aryl-1,2-Benzoquinonemonoimine and Pap=2Phenylazopyridine". Inorg. Chem, 48(20), 9800-9810.

12. De Angelis, F. (2010). Direct vs. indirect injection mechanisms in perylene dye-sensitized solar cells: A DFT/TDDFT investigation. Chem. Phys. Lett., 493, 323-327.

13. Fan, W., Tan, D., \& Deng, W. Q. (2012). Acene modified triphenylamine dyes for dyesensitized solar cells: a computational study. ChemPhysChem, 13, 2051-2060.

14. Foresman, J. B., \& Frisch, Æ. (1996). Exploring Chemistry with Electronic Structure Methods. Pittsburgh.: Second Edition, Gaussian Inc. 
15. Frisch, M., Trucks, G., Schlegel, H., Scuseria, G., Robb, M., Cheeseman, J., . . . Pople, J. A. (2004). Gaussian 03, Revision E.01. Gaussian. Wallingford C. T.: Inc.

16. Fukui, K. (1975.). Theory of Orientation. Springer.

17. Fukui, K., Yonezawa, T., \& Shingu, H. (1952). The Journal of Chemical Physics, 20, 722.

18. Geerlings, P., De Proft, F., \& Langenaeker, W. (2003). Conceptual Density Functional Theory. Chem. Rev., 103(5), 1793-1874.

19. Grätzel, M. (2009). Recent advances in sensitized mesoscopic solar cells. Acc. Chem. Res., 42, 1788-1798.

20. Guo, P., Ma, R., Guo, L., Yang, L., Liu, J., Zhang, X., . . . Dai, S. (2010). J. Mol. Graph. Mod, 29, 498-505.

21. Hagfeldt, A., \& Gratzel, M. (1995). Light-Induced Redox Reactions in Nanocrystalline Systems. Chem. Rev., 95, 49-68.

22. Ham, H. W., \& Kim, Y. S. (2010). Theoretical study of indoline dyes for dye-sensitized solar cells. Thin Solid Films, 518(21), 6558-6563.

23. Han, L. H., Zhang, C. R., Zhe, J. W., Jin, N. Z., Shen, Y. L., Wang, W., . . . Liu, Z. J. (2013). Understanding the electronic structures and absorption properties of porphyrin sensitizers YD2 and YD2-o-C8 for dye-sensitized solar cells. Int. J. Mol. Sci., 14, 20171-20188.

24. Hotze, A. C., Koojman, H., Anthony, L., Spek, J., Haasnoot, G., \& Reedijk, J. (2004). Synthesis and characterization of ruthénium (II) complexes with the new ligand 2-phenylazopyridine-5-sulfonic acid (Hsazpy) in search of new anticancer agents. New. J. Chem., 28, 565569.

25. Islam, A., Sugihara, H., \& Arakawa, H. (2003). Molecular design of ruthenium(II) polypyridyl photosensitizers for efficient nanocrystalline TiO2 solar cells. J. Photochem. Photobiol. A, 158, 131-138.

26. Koopmans, T. (1934). Uber die Zuordnung von Wellenfunktionen und Eigenwerten zu den einzelnen Elektronen eines Atoms. Physica, 1, 104-113.

27. Krause, R. A., \& Krause, K. (1980). Chemistry of bipyridyl-like ligands. Isomeric complexes of ruthenium(II) with 2(phenylazo)pyridine. Inorg. Chem, 19, 2600-2603.

28. Li, M., Kou, L., Diao, L., Zhang, Q., Li, Z., Wu, Q., .. . Wei, Z. (2015). Theoretical study of WS-9-Based organic sensitizers for unusual vis/NIR absorption and highly efficient dye-sensitized solar cells. $J$. Phys. Chem., 119, 9782-9790.

29. Marcus, R. A. (1993). Electron transfer reactions in chemistry. Theory and experiment. Rev. Mod. Phys., 65, 599-610. 
30. Nazeeruddin, M. K., Humphry-Baker, R., Liska, P., \& Grätzel, M. (2003). Inverstigation of sensitizer adsorption and influence of protons on current and voltage of dye-sensitized nanocrystalline $\mathrm{TiO} 2$ solar cell. J. Phys. Chem. B, 107, 8981-8987.

31. N'guessan, K. N., Bamba, K., Ouattara, W. P., \& Ziao, N. (2017). Theoretical investigation of the Structure Activity Relationships (SARs) of a series of five isomeric $\alpha, \beta, \gamma, \delta, \varepsilon$ ruthenium complexes $\mathrm{RuCl} 2 \mathrm{~L} 2$ with azopyridine ligands [L= azpy, tazpy, 4mazpy, 5mazpy]. Int. Journal of Engineering Research and Application, 7, 58-70.

32. Nobel, N. K., Bamba, K., Patrice, O. W., \& Ziao, N. (2017). NBO Population Analysis and Electronic Calculation of Four Azopyridine Ruthenium Complexes by DFT Method. Computational Chemistry(5), 51-64.

33. O’Regan, B., \& Graetzel, M. (1991). A Low-Cost, High-Efficiency Solar Cell Based on Dye Sensitized Colloidal TiO2 Films. Nature, $353,737-740$.

34. Preat, J., Michaux, C., Jacquemin, D., \& Perpete, E. A. (2009). Enhanced efficiency of organic dye-sensitized solar cells: triphenylamine derivatives. J. Phys. Chem. C, 113, 16821-16833.

35. Qin, C., \& Clark, A. E. (2007). DFT characterization of the optical and redox properties of natural pigments relevant to dye-sensitized solar cells. Chem. Phys. Lett., 26, 438.

36. Ruiz-Anchondo, T., Flores-Holguín, N., \& Glossman-Mitnik, D. (2010). Natural carotenoids as nanomaterial precursors for molecular photovoltaics: A computational DFT study. Molecules, 15(23), 44904510 .

37. Shalabi, A. S., El Mahdy, A. M., Assem, M. M., Taha, H. O., \& Soliman, A. K. (2014). theoretical characterization of highly efficient porphyrazin dye sensitized solar cells. J Nanopart Res, 16, 2579.

38. Shalabi, A., El Mahdy, A., Taha, H., \& Soliman, K. (2015). The effects of macrocycle and anchoring group replacements on the performance of porphyrin based sensitizer: DFT and TD-DFT study. J. Phys. Chem. Solids, 76, 22-33.

39. Shriver, D. F., \& Atkins, P. (1999). Inorganic Chemistry, 3rd Edition. New York: Oxford University Press.

40. Smedad, G., Bignozzi, C. A., \& Argazzi, R. (1994). Testing of dye sensitizedTiO2 solar cells I: Experimental photocurrent output and conversion efficiencies. Sol. Energy Mat. Sol. Cells.(32), 259.

41. Tiam-Huey, L., Tarun, K. M., Po-Ching, L., Fen-Ling, L., \& ChungSun, C. (2003). Synthesis and X-ray characterization of two isomeric dichloro bis-\{1-(phenylazo)isoquinoline $\}$ complexe of ruthénium (II). Polyhedron , 22 , 535-541. 
42. Torrent-Sucarrat, M., De Proft, F., Geerlings, P., \& Ayers, P. W. (2008). Do the local softness and hardness indicate the softest and hardest regions of a molecule? Chem Eur. J., 14, 8652-8660.

43. Velders, A. H., Schilden, K. v., Hotze, A. A., Reedijk, J., Kooijman, H., \& Spek, A. L. (2004). Dichlorobis(2-phenylazopyri dine)ruthenium(II) complexes: characterisation, spectroscopic and structural properties of four isomers. Dalton Trans, 448-455.

44. Velders, A. H., van der Schilden, K., Hotze, A. A., Reedijk, J., Kooijman, H., \& Spek, A. L. (2004). Dichlorobis(2-phenylazopyri dine)ruthenium(II) complexes: characterisation, spectroscopic and structural properties of four isomers. Dalton Trans., 448-455.

45. Xue-Feng, R., Jun, Z., \& Guo-Jun, K. (2015). Theoretical studies of electronic structure and photophysical properties of a series of indoline dyes with triphenylamine ligand. journal of nanomaterials.

46. Zhang, C. R., Liu, Z. J., Chen, Y. H., Chen, H. S., Wu, Y. Z., Feng, W., \& Wang, D. B. (2010). Curr. Appl. Phys, 10, 77.

47. Zhang, C. R., Liu, Z. J., Sun, Y. T., Shen, Y. L., Chen, Y. H., Liu, Y. J., \& Zhang, H. (2011). Spectrochim. Acta A, 79, 1843-1848.

48. Zhang, C., Liu, Z., Chen, Y., Chen, H., Wu, Y., \& Yuan, L. (2009). DFT and TDDFT study on organic dye sensitizers D5, DST and DSS for solar cells. J. Mol. Struct. THEOCHEM, 899(20), 86-93. 